\title{
Discontinuity of the Wilson String Tension in the 4-Dimensional Lattice Pure Gauge Potts Model
}

\author{
L. Laanait *, A. Messager, and J. Ruiz \\ Centre de Physique Théorique ${ }^{\star \star}$, CNRS-Luminy, Case 907, F-13288 Marseille Cedex 9, France
}

\begin{abstract}
We consider the 4-dimensional $q$-state pure gauge Potts model. For $q$ large enough, we give a new proof of the existence of a unique coupling constant $\beta_{t}$, where a first order phase transition occurs. Moreover we prove the following new results: The string tension is discontinuous at $\beta_{t}$, the Wilson parameter exhibits at $\beta_{t}$ a direct transition from an area law decay (quark confinement) to a perimeter law decay (quark deconfinement).
\end{abstract}

\section{Introduction}

The $q$-state Potts lattice gauge model was introduced by Kogut [8], according to Wilson's formulation of gauge theories on a lattice [9]. This model was considered as a natural extension of the Ising lattice gauge model, treated by several authors (see [10] and references therein). Still now there exist a great deal of rigorous results on the deconfining phase transition for the pure gauge Ising model in dimension $d \geqq 3[11,12]$ and for the $U(1)$ model defined with the Wilson's action in [14-16]. Also Monte-Carlo experiments were proposed for the Ising gauge model in $[17,18]$.

Concerning the 4-dimensional Potts pure gauge model there were some previous results on its phase diagram in [8]. An improvement of the results obtained in [8] was suggested in [19] by using a perturbative cluster expansion. However topological problems inherent to the 4- $d$ Potts gauge model were not elucidated, this was emphasized by Aizenman and Fröhlich in [20].

In both its scalar and gauge formulations the Potts model gives rise to great investigations. For $q$ large enough it exhibits a first order phase transition either in dimension $d \geqq 2$ for the scalar model or for the gauge model in dimension $d \geqq 3$. Namely, there exists a transition point where ordered phases coexist with a "disordered" one as proved in [1] by using Reflexion positivity [2]. Later a

\footnotetext{
* Université d'Aix-Marseille II, Faculté des Sciences de Luminy (On leave from Ecole Normale Supérieure, Rabat, Morocco)

$\star \star$ Laboratoire propre LP. 7061, Centre National de la Recherche Scientifique
} 
generalization of the Pirogov-Sinai theory [4] contains - in particular - similar results for the scalar model. Using the approach proposed in [5] Martirosian [6] proved that there exist only $(q+1)$ phases at the transition point for the scalar model in dimension $d \geqq 2$. Using quite a different method the phase coexistence and the surface tensions between coexisting phases were analysed for $d=2$ in [3].

In this article we derive previous results about the existence of a first order phase transition between the ordered phase and the disordered one. Moreover the approach we use enables us to study the behaviour of the Wilson loop with respect to boundary conditions.

This approach is based on the method used in [3]. It consists of observing that a partition function, at coupling constant $\beta$, in a finite lattice with the "free" boundary condition is transformed by the duality, up to a factor, into a partition function, at coupling constant $\beta^{*}$ dual of $\beta$, in the dual lattice with the "ordered" boundary condition. At the self dual point determined by the equation $\beta=\beta^{*}$ this duality operation reduces to transform the "free" boundary condition into the "ordered" one of the same model, by paying a factor proportional to the boundary. More precisely the duality transformation will be used to verify the Peierls-type condition [4] for ordered boundary conditions in both original and dual lattices. This first enables us to prove that the ordered phase and the disordered one coexist, at the transition point $\beta_{t}=\log (\sqrt{q}+1)$ determined by the equation $\beta=\beta^{*}$, as conjectured in [8]. Secondly we prove that the Wilson parameter satisfies the two following behaviours:

- For $\beta \leqq \beta_{t}$ it exhibits an area law decay with a strictly positive string tension. - For $\beta>\beta_{t}$ it exhibits a perimeter law decay with a bounded self energy.

This way of using the duality transformation as a "symmetry" of the partition function allows us to treat the self dual models (2- $d$ spin model and 4- $d$ gauge model). However, combined with the standard Pirogov-Sinaï theory the non-self dual model as the 3-d ones can be studied. This is the subject of [7], where in particular the direct transition from perimeter law to area law for the 3-d gauge model is shown. This kind of transition was previously studied in a system of random surface by Aizenman et al. [29] who showed that the two phases of the 3-d plaquettes system are in precise correspondence with the two phases of the dual system of random bonds; thus under the hypothesis that this last transition is sharp, then the former will also be sharp; the sharpness of the transition of the random bonds model was previously known in two dimensions [30,31], and recently proved in $d \geqq 1$ [32].

The paper is organized as follows: In Sect. 2, we give the definitions and state our main results, and in Sect. 3 we introduce the duality transformation of the model; we use the cell complex formalism which is very useful in our case; it is summarized for the convenience of the reader in Appendix A.1 and A.2. The proof of the first theorem (discontinuity of the free energy) is given in Sect. 4. It is based on contour estimates; to prove the "Peierls condition" we need also a geometric technical lemma whose proof is given in the Appendix B. This proof needs topological estimates, so we added further topological definitions on cell complexes in the Appendix A.3. In Sect. 5 we give a contour expansion for the partition function. The proof of the second theorem (behaviour of the Wilson parameter) which is based on contour expansions, is given in the Sect. 6 . 


\section{Definitions and Results}

\subsection{Definition of the Model}

A $G$-valued 1-cochain $\sigma \in C^{1}(K, G)$ on a complex $K$ may be interpreted as a configuration of a lattive gauge model (cf. Appendix A). Considering in particular $\mathbb{Z}_{q}$-valued cochains (we shall represent $\mathbb{Z}_{q}$ as a set of integers $\{0,1, \ldots, q-1\}$ with addition modulo $q$ as a group law), we introduce the gauge Potts model on a finite cell complex $K$ with partition function and Hamiltonian defined by ${ }^{1}$

$$
\begin{aligned}
\mathbf{Z}(K, \beta) & =\sum_{\sigma \in C^{1}(K)} e^{-\beta H_{K}(d \sigma)}, \\
H_{K}(\varphi) & =-\sum_{p \in K}^{(+)}(\delta[\varphi(p)]-1) \text { for } \varphi \in C^{2}(K),
\end{aligned}
$$

where $\delta$ is the Kronecker symbol: $\delta[\alpha]=1$ if $\alpha=0$ in $\mathbb{Z}_{q}$ and $\delta[\alpha]=0$ otherwise. The sum in (2.2) is only over positively oriented 2-cells (plaquettes). Let us remark that (2.2) is used in (2.1) with $\varphi=d \sigma$, where $d \sigma(p)=\sigma(\partial p)$. Here the differential operator $d$ and the boundary operator $\partial$ are restricted to the complex $K$ (a cell subcomplex of the cell complex associated with the lattice $\mathbb{Z}^{4}$ and denoted by $\mathbb{L}$ ); this is actually $a$ way of introducing certain boundary conditions. In particular if $K$ is closed (respectively open) $\mathbf{Z}(K, \beta)$ is a partition function with the free (respectively ordered " 0 ") boundary condition. We introduce as usual the free energy:

$$
F(\beta)=\operatorname{Lim}_{\boldsymbol{K} \uparrow \mathbb{L}} \frac{1}{N^{0}(K)} \log \mathbf{Z}(K, \beta),
$$

where $N^{p}(K)$ is the number of positively oriented $p$-cells in $K$.

Remark. The Hamiltonian $H(d \sigma)$ in (2.1) is invariant under the gauge transformation

$$
\sigma \rightarrow \sigma^{\prime}=\sigma+d \omega, \omega \in C^{0}(K) .
$$

Namely $H(d \sigma)$ is constant on the group of $\mathbb{Z}_{q}$-valued cocycles $Z^{1}(K)$ and may be expressed as function on the $\mathbb{Z}_{q}$-valued coboundary group $B^{2}(K)$. Let $|G|$ denote the cardinality of $G$, we introduce the gauge fixed (g.f.) partition function:

$$
\mathbf{Z}^{\text {g.f. }}(K, \beta)=\sum_{b \in B^{2}(K)} e^{-\beta H_{K}(b)}=\frac{\mathbf{Z}(K, \beta)}{\left|Z^{1}(K)\right|},
$$

where

$$
\left|Z^{1}(K)\right|=\frac{C^{1}(K)}{\left|B^{2}(K)\right|}=\frac{q^{N^{1}(K)}}{\left|B^{2}(K)\right|},
$$

since $C^{1}(K)$ is the direct sum of $Z^{1}(K)$ and a group isomorphic to $B^{2}(K)$.

\footnotetext{
${ }^{1}$ Since we shall only consider $\mathbb{Z}_{q}$-valued cochains, to simplify the notations we drop hereafter the corresponding specification and denote $C^{P}(K)$ instead of $C^{P}\left(K, \mathbb{Z}_{q}\right)$ and analogously for its subgroups
} 
For every $g$ we introduce its expectation value with respect to the conditional Gibbs measure:

$$
\begin{gathered}
\mu(\sigma)=(\mathbf{Z}(K, \beta))^{-1} e^{-\beta H_{K}(d \sigma)}, \\
\langle g(\sigma)\rangle_{K}^{\text {b.c. }}(\beta)=(\mathbf{Z}(K, \beta))^{-1}[g(\sigma)]_{K}^{\text {b.c. }}(\beta)=(\mathbf{Z}(K, \beta))^{-1} \sum_{\sigma \in \mathbf{C}^{1}(K)} g(\sigma) e^{-\beta H_{K}(d \sigma)} .
\end{gathered}
$$

Let $\langle\cdot\rangle^{0}(\beta)$ and $\langle\cdot\rangle^{f}(\beta)$ denote respectively the infinite volume limits of $\langle\cdot\rangle_{K}^{0}(\beta)$ and $\langle\cdot\rangle_{K}^{f}(\beta)$ when $K \uparrow \mathbb{L}$. These limits exist by correlation inequalities. Among the interesting expectations we shall consider the expectation of a plaquette observable $\langle\delta[d \sigma(p)]\rangle_{K}^{\text {b.c. }}(\beta)$, and the expectation of the Wilson loop defined as follows: Let $R$ be a rectangle of size of length $L$ and $T$ and $\mathscr{S}_{0}$ be the integral 2-chain which takes value 1 on the positively oriented plaquettes of $R$ and zero otherwise. We let $\mathscr{L}$ denote the integral 1 -cycle (loop) boundary of $\mathscr{S}_{0}: \mathscr{L}=\partial \mathscr{S}_{0}$. It satisfies for any $\sigma \in C^{1}(K)$ :

$$
d \sigma\left(\mathscr{S}_{0}\right)=\sigma(\mathscr{L})=\sum_{b \in K}^{(+)} \sigma(b) \mathscr{L}(b)=\sum_{p \in K}^{(+)} d \sigma(p) \mathscr{S}_{0}(p),
$$

where $b$ denote a 1 -cell (bond) of $K$. We successively define the Wilson parameter $W_{\mathscr{L}}^{\text {b.c. }}(\beta)[20]$, the Wilson string tension and the self-energy [10] by:

$$
\begin{aligned}
W_{\mathscr{L}}^{\text {b.c. }}(\beta) & =\frac{1}{q-1}\langle q \delta[\sigma(\mathscr{L})]-1\rangle^{\text {b.c. }}(\beta)=\frac{1}{q-1} \sum_{\alpha=1}^{q-1}\left\langle e^{\frac{2 i \pi}{q} \alpha \sigma(\mathscr{L})}\right\rangle^{\text {b.c. }}(\beta), \\
s(\beta) & =-\operatorname{Lim}_{L T \uparrow \infty} \frac{1}{L \cdot T} \log W_{\mathscr{L}}^{f}(\beta), \\
\tau(\beta) & =-\operatorname{Lim}_{L T \uparrow \infty} \frac{1}{L+T} \log W_{\mathscr{L}}^{0}(\beta) .
\end{aligned}
$$

\subsection{Results}

Our main results are contained in the following theorems:

Theorem 2.1. The 4-dimensional pure gauge model exhibits, whenever $q$ is large enough, a first order phase transition at $\beta_{t}(q)=\log (\sqrt{q}+1)$, where the derivative of the free energy with respect to $\beta$ is discontinuous:

$$
\begin{aligned}
& \left\langle\delta[d \sigma(p)]-\frac{\lambda}{2}\right\rangle^{0}\left(\beta_{t}\right)>0 \\
& \left\langle\delta[d \sigma(p)]-\frac{\lambda}{2}\right\rangle^{f}\left(\beta_{t}\right)<0, \text { with } \lambda=1+\frac{1}{\sqrt{q}} .
\end{aligned}
$$

Theorem 2.2. The Wilson parameter exhibits at $\beta_{t}$ a direct transition from an area law decay (quark confinement) to a perimeter law decay (quark deconfinement):
a) $W_{\mathscr{L}}^{f}(\beta) \leqq e^{-(k \log q-\mu) T \cdot L} \quad$ if $\beta \leqq \beta_{t}(q)$,
b) $W_{\mathscr{L}}^{0}(\beta) \geqq e^{-k^{\prime}(T+L)} \quad$ if $\quad \beta \geqq \beta_{t}(q)$,
c) $W_{\mathscr{L}}^{f}(\beta) \geqq e^{-k^{\prime}(T+L)} \quad$ if $\quad \beta>\beta_{t}(q)$,

where $k$ and $k^{\prime}$ are strictly positive constants and $\mu$ is a positive geometric constant. 
Hence the Wilson string tension is discontinuous at $\beta_{t}$ and the self-energy is bounded, above $\beta_{t}$ :

d) $s(\beta)>k$ if $\beta \leqq \beta_{t}(q)$ and $s(\beta)=0 \quad$ if $\quad \beta>\beta_{t}(q)$,

e) $\tau(\beta)=\infty$ if $\beta<\beta_{t}(q)$ and $\tau(\beta) \leqq k^{\prime} \quad$ if $\beta \geqq \beta_{t}(q)$.

\section{Duality Transformation}

The duality transformation takes into account the homological properties of the complex on which the system is defined. Let us define the following partition functions:

$$
\begin{aligned}
\Xi^{\text {g.f. }}(K, \beta) & =\sum_{z \in Z^{2}(K)} e^{-\beta H_{K}(z)}, \\
\Xi(K, \beta) & =\left|Z^{1}(K)\right| \Xi^{\text {g.f. }}(K, \beta) .
\end{aligned}
$$

Proposition 3.1. a) The partitions functions in a complex $K \subset \mathbb{L}$ and in its dual $K^{*} \subset \mathbb{L}^{*}$ satisfy:

$$
\mathbf{Z}(K, \beta)=\left(\frac{e^{\beta}-1}{q}\right)^{N^{2}(K)} e^{\left(\beta^{*}-\beta\right) N^{2}(K)} q^{N^{1}(K)} \Xi^{\text {g.f. }}\left(K^{*}, \beta^{*}\right),
$$

where the dual coupling $\beta^{*}$ is given by:

$$
\left(e^{\beta^{*}}-1\right)\left(e^{\beta}-1\right)=q .
$$

b) Whenever the $\mathbb{Z}_{q}$-valued 2-homology group of $K$ is trivial, $H_{2}(K)=\{0\}$, and $P$ is a subcomplex of $K$, then:

$$
\left\langle\prod_{p \in P}\left[\delta[d \sigma(p)]-\frac{\lambda}{2}\right]\right\rangle_{K}\left(\beta_{t}\right)=(-1)^{N^{2}(P)}\left\langle\prod_{p \in P^{*}}\left[\delta\left[d \sigma_{*}\left(p_{*}\right)\right]-\frac{\lambda}{2}\right]\right\rangle_{K^{*}}\left(\beta_{t}\right),
$$

where $\beta_{t}=\log (\sqrt{q}+1)$ is the self-dual coupling obtained by letting $\beta=\beta^{*}$ in (3.3). Proof. We consider the Fourier expansion of $e^{\beta \delta(\alpha)}$ (the dual group of $\mathbb{Z}_{q}$ is identified with $\mathbb{Z}_{q}$ ):

$$
\begin{aligned}
e^{\beta \delta(\alpha)} & =1+\left(e^{\beta}-1\right) \delta(\alpha)=\sum_{n=0}^{q-1}\left[\delta(n)+\frac{e^{\beta}-1}{q}\right] e^{\frac{2 i \pi}{q} n \alpha} \\
& =\frac{e^{\beta}-1}{q} \sum_{n=0}^{q-1}\left[1+\frac{q}{e^{\beta}-1}\right]^{\delta(n)} e^{\frac{2 i \pi}{q} n \alpha}=\frac{e^{\beta}-1}{q} \sum_{n=0}^{q-1} e^{\beta * \delta(n)} e^{\frac{2 i \pi}{q} n \alpha}
\end{aligned}
$$

We insert this formula for every 2 -cell in (2.2). Considering the scalar product $(\phi, \psi)$ $=\sum_{s^{p} \in K}^{(+)} \phi\left(s^{p}\right) \psi\left(s^{p}\right)$; for every two $\mathbb{Z}_{q}$-valued $p$-cochains $\phi$ and $\psi$ on $K$ we get

$$
\mathbf{Z}(K, \beta)=\left(\frac{e^{\beta}-1}{q}\right)^{N^{2}(K)} e^{\left(\beta^{*}-\beta\right) N^{2}(K)} \sum_{\phi \in C^{2}(K)} e^{-\beta^{*} H_{K}(\phi)} \sum_{\sigma \in C^{1}(K)} e^{\frac{2 i \pi}{q}(\phi, d \sigma)} .
$$

Observing that $(\phi, d \sigma)=\left(d^{*} \phi, \sigma\right)$ the summation over $\sigma$ gives the condition $d^{*} \phi=0$. Namely,

$$
\mathbf{Z}(K, \beta)=\left(\frac{e^{\beta}-1}{q}\right)^{N^{2}(K)} e^{\left(\beta^{*}-\beta\right) N^{2}(K)} q^{N^{1}(K)} \sum_{z \in \mathbf{Z}_{2}(K)} e^{-\beta^{*} H_{K}(z)}
$$


We then use the isomorphism between $\mathbf{Z}_{2}(K)$ and $\mathbf{Z}^{2}\left(K^{*}\right)$ and the mapping (A.4), to derive the statement a).

To prove the statement b) we use $\delta(\alpha) e^{\beta(\delta(\alpha)-1)}=\delta(\alpha)$ for $\alpha \in \mathbb{Z}_{q}$ and proceed as for the proof of the statement a).

Notice that at $\beta_{t}$ the Fourier transform of $\left[\delta(\alpha)-\frac{\lambda}{2}\right] e^{\beta_{t} \delta(\alpha)}$ is up to a constant given by its own negative.

Remark. Let us notice that if $K$ is open (respectively closed) then $K^{*}$ is closed (respectively open) and thus the duality transform free boundary conditions into ordered ones and conversely.

\section{Contour Estimates and Proof of Theorem 2.1}

\subsection{Definition of Contours}

To introduce contours we shall use the concepts of the envelope and of the fringe of a set $Q^{p}$ of lattice $p$-cells contained in $\mathbb{L}^{p}, Q^{p} \subset \mathbb{L}^{p}$. We will denote by $\bar{Q}^{p}$ the closure of $Q^{p}$ and we define:

- the envelope $E\left(Q^{p}\right)$ of $Q^{p}$ as the maximal closed subcomplex of $\mathbb{L}$ whose set of $r$-cells, $r \leqq p$ coincides with $\bar{Q}^{p}, E\left(Q^{p}\right) \cap \mathbb{L}^{p}=Q^{p}$. An explicit expression is $E\left(Q^{p}\right)$ $=\bigcup_{q=p+1}^{4} E^{q}\left(Q^{p}\right) \cup \bar{Q}^{p}$, with $E^{p}\left(Q^{p}\right)=Q^{p}$ and $E^{q}\left(Q^{p}\right)=\left\{s^{q} \in \mathbb{L}^{q} \mid\right.$ all $s^{q-1}$ of $\partial s^{q}$ belongs to $\left.E^{q-1}\left(Q^{p}\right)\right\}$ whenever $q \geqq p+1$.

- the fringe $F\left(Q^{p}\right)$ of $Q^{p}$ by $F\left(Q^{p}\right)=\mathbb{L} \backslash\left\{E\left(Q^{p}\right) \cup E\left(\mathbb{L}^{0} \backslash \mathbb{L}^{0} \cap E\left(Q^{p}\right)\right\}\right.$.

- the boundary $B(K)$ of a cell complex $K$ by $B(K)=\overline{\mathbb{L} \backslash K} \cap K$.

Consider now a configuration $\sigma \in C^{1}(\mathbb{L})$ such that the set of disordered plaquettes $M^{2}(\sigma)=\left\{p \in \mathbb{L}^{2} \mid \sigma(\partial p) \neq 0\right\}$ is finite. Denoting by $Q^{2}(\sigma)$ the unique infinite component (connected subcomplex) of the complex $\mathbb{L}^{2} \backslash M^{2}(\sigma)$, we shall denote by $F(\sigma)$ the fringe of $Q^{2}(\sigma): F(\sigma)=F\left(Q^{2}(\sigma)\right)$. Clearly if $p \in F(\sigma)$ implies $\sigma(\partial p) \neq 0$.

A pair $\tilde{\gamma}=\left\{\gamma, \sigma_{\gamma}\right\}$, where $\gamma$ is a component of $F(\sigma)$ and $\sigma_{\gamma}$ the restriction of $\sigma$ on the complex $\gamma$, will be called an external contour of $\sigma$. A pair $\tilde{\gamma}=\left\{\gamma, \sigma_{\gamma}\right\}$ with $\gamma$ a subcomplex of $\mathbb{L}$ and $\sigma$ a configuration on it, $\sigma \in C^{1}(\gamma)$, will be called a contour if there exists a configuration $\sigma \in C^{1}(\mathbb{L})$ with $\left|M^{2}(\sigma)\right|<\infty$ such that $\tilde{\gamma}$ is its external contour.

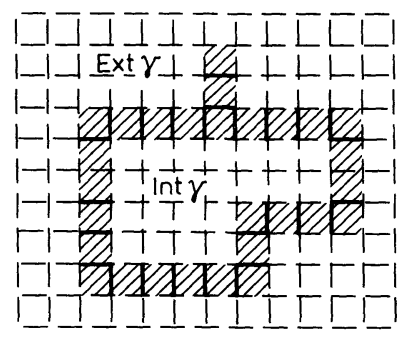

Fig. 1. The open complex $\gamma$ (black bonds and hatched plaquettes) and the closed complexes Int $\gamma$ and Ext $\gamma$; the vertice belongs to Int $\gamma$ or Ext $\gamma$ 
Whenever $\tilde{\gamma}$ is a contour, we call the complex $\gamma$ its support, $\gamma=\operatorname{supp} \tilde{\gamma}$, and introduce the complexes: Ext $\gamma$ as the unique infinite component of $\mathbb{L} \backslash \gamma, V(\gamma)$ $=\mathbb{L} \backslash \operatorname{Ext} \gamma$, and Int $\gamma=V(\gamma) \backslash \gamma$. It is easy to show that $\bar{\gamma} \cap \operatorname{Ext} \gamma=\overline{V(\gamma)} \backslash V(\gamma)$. Let us also mention that the complexes $\gamma$ and $V(\gamma)$ are open whereas the complexes Int $\gamma$ and Ext $\gamma$ are closed.

Two contours $\tilde{\gamma}_{1} \tilde{\gamma}_{2}$ with disjoint supports are called mutually compatible; they are called mutually compatible external contours if $V\left(\gamma_{1}\right) \subset \operatorname{Ext} \gamma_{2}$ and $V\left(\gamma_{2}\right) \subset \operatorname{Ext} \gamma_{1}$. It is easy to show that whenever $\widetilde{\theta}=\left\{\tilde{\gamma}_{1}, \tilde{\gamma}_{2}, \tilde{\gamma}_{3}, \ldots, \tilde{\gamma}_{n}\right\}$ is a family of mutually external contours, there exists a configuration $\sigma$ with the same set of external contours. For such a family $\widetilde{\theta}$ of external contours we shall use the notation: $\theta=\operatorname{supp} \widetilde{\theta}=\bigcup_{i} \gamma_{i}, \quad V(\theta)=\bigcup_{i} V\left(\gamma_{i}\right), \quad \operatorname{Int} \theta=V(\theta) \backslash \theta, \quad \operatorname{Ext} \theta=\mathbb{L} \backslash V(\theta), \quad$ and $\quad \operatorname{Ext}_{K} \theta$ $=K \cap \operatorname{Ext} \theta$ whenever $K \subset \mathbb{L}$.

For contours on the dual lattice $\mathbb{L}^{*}$ defined in the same way as above with $\mathbb{L}$ replaced by $\mathbb{L}^{*}$ we shall use the notation $\tilde{\gamma}_{*}=\left\{\gamma_{*}, \sigma_{* \gamma_{*}}\right\}$. Notice that $\gamma_{*}$ is a support of a contour and hence an open subcomplex of $\mathbb{L}^{*}$, while $\gamma^{*}$ is the dual of the complex $\gamma$ and it is thus a closed subcomplex of $\mathbb{L}^{*}$ for every $\tilde{\gamma}=\left\{\gamma, \sigma_{\gamma}\right\}$.

\subsection{Contour Estimates}

Let $\gamma$ be the support of a contour, its probability given ordered " 0 " boundary is:

$$
P_{K, \beta}\left(\left.\gamma\right|^{\prime \prime} 0 "\right)=\left\langle\chi_{\gamma}(d \sigma)\right\rangle_{K}^{0}(\beta) .
$$

$K$ is an open subcomplex union of the envelope and the coboundary of a rectangular box $\Lambda: K=E(\Lambda) \cup F(\Lambda), K \supset V(\gamma)$, and the characteristic function of the contour, denoting hereafter $\delta(X)=\prod_{p \in X} \delta[d \sigma(p)]$, is:

$$
\chi_{\gamma}(d \sigma)=\delta(\widetilde{E}) \prod_{p \in \gamma}(1-\delta[d \sigma(p)]),
$$

where $\widetilde{E}=\bar{\gamma}^{K} \cap \operatorname{Ext}_{K} \gamma\left(\bar{X}^{K}\right.$ denotes the closure of $X$ in $\left.K\right)$ and $d$ is restricted to $K$.

First step: Let us define the following partition function

$$
\begin{aligned}
\mathbf{Z}(K, \beta \mid \gamma) & =\sum_{\sigma \in \mathcal{C}^{1}(K)} \chi_{\gamma}(d \sigma) e^{-\beta H_{K}(d \sigma)}, \\
\left\langle\chi_{\gamma}(d \sigma)\right\rangle_{K}(\beta) & =\frac{\mathbf{Z}(K, \beta \mid \gamma)}{\mathbf{Z}(K, \beta)} .
\end{aligned}
$$

We first need the following

Lemma 4.1. Let $K$ and $I$ be two subcomplexes of $\mathbb{L}$ such that: the $\mathbb{Z}_{q}$-valued 2-cohomology group of $K$ is trivial, $H^{2}(K)=\{0\}, I$ is open and $I \subset K$, then

$$
\left(\left|Z^{1}(K)\right|\right)^{-1}[\delta(K \backslash I)]_{K}(\beta)=\Xi^{\text {g.f. }}(I, \beta) .
$$

b) Let then $f_{I}$ be a function on $C^{2}$ with support in $I_{0} C I$, then:

$$
\left(\left|Z^{1}(K)\right|\right)^{-1}\left[\delta(K \backslash I) f_{I}(d \sigma)\right]_{K}(\beta)=\sum_{z \in Z^{2}(I)} f_{I}(z) e^{-\beta H_{I}(z)} .
$$


Proof. Consider the canonical extension $i_{I}: C^{2}(I) \rightarrow C^{2}(K)$. Since $I$ is open in $K$, $d_{K} i_{I}=i_{I} d_{I}\left(d_{X}\right.$ denoting the restriction of $d$ to the complex $\left.X\right)$ and we get:

$$
i_{I} Z^{2}(I)=\left\{z \in Z^{2}(K) \mid z(p)=0 \quad \text { if } \quad p \in K \backslash I\right\} .
$$

Since $H^{2}(K)=\{0\}$ by assumption we have:

$$
i_{I} Z^{2}(I)=\left\{b \in B^{2}(K) \mid b(p)=0 \quad \text { if } \quad p \in K \backslash I\right\} .
$$

Hence

$$
\begin{aligned}
\Xi^{\mathrm{g} . f .} \cdot(I, \beta) & =\sum_{\sigma \in B^{2}(K)} e^{-\beta H_{K}(b)} \prod_{p \in K / I} \delta[b(p)] \\
& =\left(\left|Z^{1}(K)\right|\right)^{-1}[\delta(K \backslash I)]_{K}(\beta) .
\end{aligned}
$$

The proof of statement $b$ ) is analogous.

Remark. Taking $K$ as a complex associated to a rectangular box, the above lemma will also facilitate computations of partition functions on cohomologically nontrivial complexes contained in $K$. We shall use it in particular in the next sections to express partition functions in term of non-interacting contour models.

Let us now define the partition functions with disordered ("dis") boundary condition:

$$
\begin{gathered}
\Xi^{\text {g.f. }}(\gamma, \beta, \text { dis })=\sum_{z \in Z^{2}(V(\gamma))} e^{-\beta H_{V(\gamma)}(z)} \prod_{p \in \gamma}[1-\delta(z(p))], \\
\Xi(\gamma, \beta, \text { dis })=\left|Z^{1}(V(\gamma))\right| \Xi^{\text {g.f. }}(\gamma, \beta, \text { dis }) .
\end{gathered}
$$

We put $\widetilde{E}=\overline{V(\gamma)}^{K} \backslash V(\gamma)$ and apply the statement b) of Lemma 4.1 with $I=K \backslash \widetilde{E}$ and $f(\cdot)=\prod_{p \in \gamma}[1-\delta(\cdot(p))]$, to show:

$$
\mathbf{Z}(K, \beta \mid \gamma)=\left|Z^{1}(K)\right| \Xi^{\text {g.f. }}\left(K \backslash \bar{V}^{K}(\gamma), \beta\right) \Xi^{\text {g.f. }}(\gamma, \beta, \text { dis }),
$$

where we have used $K \backslash \widetilde{E}$ is a disjoint union of open subcomplexes in $K$.

Second step: We shall now need the following:

Lemma 4.2. a) $\Xi^{\text {g.f. }}(\gamma, \beta$, dis $)=g(\gamma, \beta) D(\gamma, \beta) \Xi^{\text {g.f. }}\left((\operatorname{Int} \gamma)^{*}, \beta^{*}\right)$,

$$
\begin{aligned}
& D(\gamma, \beta)=e^{-\beta N^{2}(\gamma)} e^{\left(\beta^{*}-\beta\right) N^{2}(\operatorname{Int} \gamma)}\left(\frac{e^{\beta}-1}{q}\right)^{N^{2}(\operatorname{Int} \gamma)} q^{N^{1}(V(\gamma))} \frac{\left|H^{2}(V(\gamma))\right|}{\left|Z^{1}(V(\gamma))\right|}, \\
& g(\gamma, \beta)=\frac{e^{\beta N^{2}(\gamma)} \Xi(\gamma, \beta, \operatorname{dis})}{\left|H^{2}(V(\gamma))\right| q^{N^{1}(\gamma)} \mathbf{Z}(\operatorname{Int} \gamma, \beta)} .
\end{aligned}
$$

b)

c)

$$
\begin{gathered}
0<g(\gamma, \beta) \leqq 1 . \\
D\left(\gamma, \beta_{t}\right) \leqq\left|H^{2}(V(\gamma))\right| q^{-N^{0}(V(\gamma))+N^{1}(V(\gamma))-\frac{N^{2}(V(\gamma))}{2}} .
\end{gathered}
$$

Proof. We have from the above definitions:

$$
\begin{aligned}
\Xi^{\mathrm{g} . \mathrm{f}}(\gamma, \beta, \text { dis }) & =\left(\left|Z^{1}(V(\gamma))\right|\right)^{-1} \Xi(\gamma, \beta, \text { dis }) \\
& =g(\gamma, \beta) e^{-\beta N^{2}(\gamma)} q^{N^{1}(\gamma)} \frac{\left|H^{2}(V(\gamma))\right|}{\left|Z^{1}(V(\gamma))\right|} \mathbf{Z}(\operatorname{Int} \gamma, \beta) .
\end{aligned}
$$


The proof of statement a) then follows from statement a) of Proposition 3.1. To prove statement $\mathrm{b})$ we sum over $\sigma \in C^{1}(\gamma)$ in $\Xi(\gamma, \beta$, dis) using $1-\delta \leqq 1$, we then show that for any $h \in C^{2}(\operatorname{Int} \gamma)$ :

$$
\frac{\sum_{\sigma \in C^{1}(\operatorname{Int} \gamma)} e^{-\beta H_{\operatorname{Int} \gamma}(d \sigma+h)}}{\sum_{\sigma \in C^{1}(\operatorname{Int} \gamma)} e^{-\beta H_{\operatorname{Int} \gamma}(d \sigma)}} \leqq 1,
$$

since the dual transform of the left-hand side of the above inequality is a correlation function which is less than 1 . Finally since $Z^{0}(X)=\{0\}$ whenever $X$ is open, then:

$$
\left(\left|Z^{1}(V(\gamma))\right|\right)^{-1} \leqq\left(\left|B^{1}(V(\gamma))\right|\right)^{-1}=q^{-N^{0}(V(\gamma))},
$$

from which statement c) follows.

We then obtain from (4.3) and Lemma 4.2:

$$
\mathbf{Z}\left(K, \beta_{t} \mid \gamma\right)=g\left(\gamma, \beta_{t}\right) D\left(\gamma, \beta_{t}\right)\left|Z^{1}(K)\right| \Xi^{\text {g.f. }}\left(K \backslash \bar{V}^{K}(\gamma), \beta_{t}\right) \Xi^{\text {g.f. }}\left((\operatorname{Int} \gamma)^{*}, \beta_{t}\right) .
$$

Third step:

Proposition 4.1. At the self-dual temperature $\beta_{t}$ the probability of a contour given ordered " 0 " boundary condition satisfies:

$$
P_{K, \beta_{t}}(\gamma \mid \text { “" } 0 ") \leqq\left|H^{2}(V(\gamma))\right| q^{-N^{0}(V(\gamma))+N^{1}(V(\gamma))-\frac{N^{2}(V(\gamma))}{2}} .
$$

Proof. We shall compare the product of the two partition function in the righthand side of (4.4) with $\mathbf{Z}(K, \beta)$. Let $T$ be the complex $(\operatorname{Int} \gamma)^{*}$ translated by $\frac{1}{2}$ in all the positive directions. From statement a) of Lemma 4.1 we deduce:

$$
\begin{aligned}
\mathbf{Z}(K, \beta) & \geqq\left[\delta\left(K \backslash\left\{T \cup\left[K \backslash \bar{V}^{K}(\gamma)\right]\right\}\right)\right]_{K}(\beta) \\
& =\left|Z^{1}(K)\right| \Xi^{\text {g.f. }}\left(T \cup\left[K \backslash \bar{V}^{K}(\gamma)\right], \beta\right) \\
& =\left|Z^{1}(K)\right| \Xi^{\text {g.f. }}(T, \beta) \Xi^{\text {g.f. }}\left(K \backslash \bar{V}^{K}(\gamma), \beta\right) .
\end{aligned}
$$

Therefore at $\beta_{t}$ the probability of a contour $\gamma$ is bounded above by $g\left(\gamma, \beta_{t}\right) D\left(\gamma, \beta_{t}\right)$ and thus the proof follows from statements b) and c) of Lemma 4.2.

Fourth step: We finally need the following:

Lemma 4.3. Whenever $\gamma$ is the support of a contour, then:

$$
\left|H^{2}(V(\gamma))\right| q^{-N^{\mathrm{o}}(V(\gamma))+N^{1}(V(\gamma))-\frac{N^{2}(V(\gamma))}{2}} \leqq q^{-\frac{N^{0}\left(\gamma^{*}\right)}{40}} .
$$

Proof. We postpone the proof to Appendix B.

Our main estimate follows from Proposition 4.1 and Lemma 4.3:

Proposition 4.2. At the self-dual temperature $\beta_{t}$ the probability of a contour given ordered " 0 " boundary condition satisfies:

$$
P_{K, \beta_{t}}(\gamma \mid “ 0 ”) \leqq q^{-\frac{N^{0}\left(\gamma^{*}\right)}{40}}
$$




\subsection{Proof of Theorem 2.1}

Let $p$ be some 2 -cell in $K=E(\Lambda) \cup F(\Lambda)$, where $\Lambda$ is a rectangular box, then:

$$
\langle\delta[d \sigma(p)]\rangle_{K}^{0}(\beta)=\operatorname{Prob}\left\{d \sigma(p)=\left.0\right|^{\prime \prime} 0 ”\right\}=1-\operatorname{Prob}\{d \sigma(p) \neq 0 \mid “ 0 ”\} .
$$

If one considers a configuration $\sigma \in C^{1}(K)$ such that $p$ is disordered, then there exists necessarily a contour $\gamma$ in such a configuration such that $\gamma$ contains or encloses the plaquette $p$, hence

$$
\operatorname{Prob}\{d \sigma(p) \neq 0 \mid “ 0 ”\} \leqq \sum_{\gamma: p \in V(\gamma)} P_{K, \beta}(\gamma \mid “ 0 ”) .
$$

We take into account Proposition 4.2 and we use standard arguments to prove that, for $q$ large enough,

$$
\langle\delta[d \sigma(p)]\rangle^{0}\left(\beta_{t}\right) \geqq 1-\varepsilon(q)>\frac{1}{2}+\frac{1}{2 \sqrt{q}} .
$$

The second formula of Theorem 2.1 is a consequence of statement b) of Proposition 3.1. This ends the proof of Theorem 2.1 since the expectations of $\delta[d \sigma(p)]$ with free and ordered boundary conditions are respectively the left and right derivative with respect to $\beta$ of the free energy $F(\beta)[21]$.

Remark. We think that with this method, more general boundary conditions can be studied, and that an estimate such as those of Proposition 4.2 can be obtained for the weight of open contours; then the set of translation invariant states should be studied with the method of [25] and one can certainly prove that, since noneven correlation functions are zero, every translation invariant state is at $\beta_{t}$ a linear combination of the two extremal states $\langle\cdot\rangle^{0}\left(\beta_{t}\right)$ and $\langle\cdot\rangle^{f}\left(\beta_{t}\right)$.

\section{Contour Expansions}

In this section we will expand the partition functions in terms of external contours in both $\mathbb{L}$ and $\mathbb{L}^{*}$ :

Lemma 5.1. Let $V$ be an open subcomplex of $\mathbb{L}$ then:

$$
\Xi^{\text {g.f. }}(V, \beta)=\sum_{\theta \subset V} \Xi^{\text {g.f. }}(\theta, \beta, \text { dis }),
$$

where the sum is over all supports of families of mutually external contours satisfying $V(\theta) \cap \mathbb{L}^{1} \subset V$.

Proof. Let $K$ be the envelope of a rectangular box $\Lambda$ such that $K=E(\Lambda) \supset V$, and let:

$$
\chi_{\theta ; K}=\delta(K \backslash V(\theta)) \prod_{p \in \theta}(1-\delta[d \sigma(p)]),
$$

where the boundary operator is restricted to $K$. It is clear that we can write

$$
[\delta(K \backslash V)]_{K}(\beta)=\sum_{\theta \subset V}\left[\chi_{\theta ; K}\right]_{K}(\beta) .
$$


From statement b) of Lemma 4.1 it follows that

$$
\begin{aligned}
& {[\delta(K \backslash V)]_{K}(\beta)=\left|Z^{1}(K)\right| \Xi^{\text {g.f. }}(V, \beta),} \\
& {\left[\chi_{\theta, K}\right]_{K}(\beta)=\left|Z^{1}(K)\right| \Xi^{\text {g.f. }}(\theta, \beta, \text { dis }),}
\end{aligned}
$$

and we then deduce the result.

From Lemmas 5.1 and 4.2 it follows for open complexes $V \subset \mathbb{L}$ and $V_{*} \subset \mathbb{L}^{*}$ :

$$
\begin{gathered}
\Xi^{\text {g.f. }}(V, \beta)=\sum_{\theta \subset V} \Xi^{\text {g.f. }}(\theta, \beta, \text { dis }), \\
\Xi^{\text {g.f. }}(\gamma, \beta, \text { dis })=g(\gamma, \beta) D(\gamma, \beta) \Xi^{\text {g.f. }}\left((\operatorname{Int} \gamma)^{*}, \beta^{*}\right), \\
\Xi^{\text {g.f. }}\left(V_{*}, \beta^{*}\right)=\sum_{\theta_{*} \subset V_{*}} \Xi^{\text {g.f. }}\left(\theta_{*}, \beta^{*}, \text { dis }\right), \\
\Xi^{\text {g.f. }}\left(\gamma_{*}, \beta^{*}, \operatorname{dis}\right)=g\left(\gamma_{*}, \beta^{*}\right) D\left(\gamma_{*}, \beta^{*}\right) \Xi^{\text {g.f. }}\left(\left(\operatorname{Int} \gamma_{*}\right)^{*}, \beta\right) .
\end{gathered}
$$

For $\theta$ a family of mutually external contours, we have

$$
\Xi^{\text {g.f. }}(\theta, \beta, \text { dis })=\prod_{\gamma \in \theta} \Xi^{\text {g.f. }}(\gamma, \beta, \text { dis }) .
$$

Proposition 5.1. We let $D(V)$ the set of supports of families of mutually compatible contours in $V$ and we define:

where $\stackrel{\circ}{X}=X \backslash B(X)$ then:

$$
\phi(\gamma)=\frac{\Xi^{\text {g.f. }}\left(\gamma, \beta_{t}, \text { dis }\right)}{\Xi^{\text {g.f. }}\left(\operatorname{Int} \gamma, \beta_{t}\right)},
$$

a) $\Xi^{\text {g.f. }}\left(V, \beta_{t}\right)=\sum_{\partial \in D(V)} \prod_{\gamma \in \partial} \phi(\gamma)$.

b) Whenever $q$ is large enough, $\phi$ is a $\tau$-functional:

$$
0<\phi(\gamma) \leqq e^{-\tau N^{0}\left(\gamma^{*}\right)}, \text { where } \tau \geqq k_{0} \log q, k_{0}>0 .
$$

Proof. To prove statement a) we use Lemma 5.1 and (5.2), and iterate on $\Xi^{\text {g.f. }}\left(\right.$ Int $\left.\gamma, \beta_{t}\right)$. For statement b) we first notice:

$$
\phi(\gamma) \leqq D\left(\gamma, \beta_{t}\right) \frac{\Xi^{\text {g.f. }}\left((\operatorname{Int} \gamma)^{*}, \beta_{t}\right)}{\Xi^{\text {g.f. }}\left(\operatorname{Int} \gamma, \beta_{t}\right)} .
$$

We let $T$ be the complex $(\operatorname{Int} \gamma)^{*}$ translated by $+\frac{1}{2}$ in all positive directions, from statement a) of Lemma 4.1 we get:

$$
\frac{\Xi^{\text {g.f. }}(\operatorname{Int} \gamma, \beta)}{\Xi^{\text {g.f. }}\left((\operatorname{Int} \gamma)^{*}, \beta\right)}=\frac{[\delta(K \backslash \operatorname{Int} \gamma)]_{K}(\beta)}{[\delta(K \backslash T)]_{K}(\beta)},
$$

where $K \supset T$ and $H^{2}(K)=\{0\}$. We then use

$$
\delta(K \backslash \operatorname{In} \mathrm{\circ} \gamma) e^{-\beta H_{\operatorname{In} t}}=\delta(K \backslash T) \delta(T \backslash \operatorname{Int} \gamma) e^{-\beta H_{T}}
$$

and $\lim _{\beta \rightarrow \infty} e^{\beta(\delta-1)}=\delta$ to obtain by correlation inequalities $([22])$ :

$$
\begin{aligned}
\frac{\Xi^{\text {g.f. }}\left(\operatorname{Int} \gamma, \beta_{t}\right)}{\Xi^{\text {g.f. }}\left((\operatorname{Int} \gamma)^{*}, \beta_{t}\right)} & \geqq\langle\delta(T \backslash \operatorname{Int} \gamma)\rangle_{K}\left(\beta_{t}\right) \\
& \geqq\left[\langle\delta(T \backslash \text { Int } \gamma)\rangle_{K}^{0}\left(\beta_{t}\right)\right]^{N^{2}(T \backslash \text { Int } \gamma)} .
\end{aligned}
$$


Since we are free to choose $K$ open this is greater than $\left(\frac{\lambda}{2}\right)^{k^{\prime} N^{0}\left(\gamma^{*}\right)}$ by Theorem 2.1. The $\tau$-functionality then follows from statement c) of Lemmas 4.2 and 4.3 .

Remark. One can also obtain contours expansion at $\beta \neq \beta_{t}$. We think that using the method of [28], one can prove that for $\beta \neq \beta_{t}$ there is a unique Gibbs state, since non-even correlation functions are zero: the free energy would be differentiable with respect to $\beta$, and the free state should coincide with the ordered one.

\section{Proof of Theorem 2.2}

\subsection{Proof of Statements b) and c) of Theorem 2.2: Perimeter Law Decay}

Our proof is based on a contour expansion of $[q \delta[\sigma(\mathscr{L})]-1]_{K}^{0}(\beta)$, where $K$ is an open subcomplex of $\mathbb{L}$. We assume $K=E(\Lambda) \cup F(\Lambda)$, where $\Lambda$ is a rectangular box, and $K$ contains the complex $\left\{\mathscr{S}_{0}\right\}$ consisting of the plaquettes, bonds and sites of this surface. For convenience let us define the following "modified" partition function for an open subcomplex $V$ of $K$ :

$$
\Xi_{\alpha}^{\mathrm{g} . \mathrm{f} .}(V, \beta)=\sum_{z \in Z^{2}(V)} e^{\frac{2 i \pi}{q} \alpha z\left(\mathscr{S}_{\mid V}\right)} e^{-\beta H_{V}(z)} .
$$

$\mathscr{S}$ is an integral 2-chain satisfying $\partial \mathscr{S}=\mathscr{L}$ and $\mathscr{P}_{\mid V}$ is the restriction to $V$ of $\mathscr{S}$. Let us remark that:

$$
\langle q \delta[\sigma(\mathscr{L})]-1\rangle_{K}^{0}(\beta)=\sum_{\alpha=1}^{q-1} \frac{\Xi_{\alpha}^{\text {g.f. }}(K, \beta)}{\Xi^{\text {g.f. }}(K, \beta)},
$$

since the cohomology group $H^{2}(K)$ is trivial.

Lemma 6.1. Let $V$ be an open subcomplex of $K$ then:

$$
\Xi_{\alpha}^{\text {g.f. }}(V, \beta)=\sum_{\theta \subset V} \Xi_{\alpha}^{\text {g.f.f. }}(\theta, \beta, \text { dis }),
$$

where the sum is over all supports of families of mutually external contours satisfying $V(\theta) \cap \mathbb{L}^{1} \subset V$ and

$$
\Xi_{\alpha}^{\text {g.f. }}(\theta, \beta, \text { dis })=\sum_{z \in Z^{2}(V(\theta))} e^{-\beta H_{V(\theta)}(z)} e^{\frac{2 i \pi}{q} \alpha z\left(\mathscr{S}_{\mid V(\theta)}\right)} \prod_{p \in \theta}[1-\delta(z(p))] .
$$

Proof. We define $\chi_{\theta ; K}$ as in (5.1) and get:

$$
\left[\delta(K \backslash V) e^{\frac{2 i \pi}{q} \alpha d \sigma(\mathscr{S})}\right]_{K}(\beta)=\sum_{\theta \subset V}\left[\chi_{\theta ; K} e^{\frac{2 i \pi}{q} \alpha d \sigma(\mathscr{S})}\right]_{K}(\beta) .
$$

We use statement b) of Lemma 4.1 to deduce:

$$
\begin{gathered}
{\left[\delta(K \backslash V) e^{\frac{2 i \pi}{q} \alpha d \sigma(\mathscr{S})}\right]_{K}(\beta)=\left|Z^{1}(K)\right| \Xi_{\alpha}^{\text {g.f. }}(V, \beta),} \\
{\left[\chi_{\theta ; K} e^{\frac{2 i \pi}{q} \alpha d \sigma(\mathscr{S})}\right]_{K}(\beta)=\left|Z^{1}(K)\right| \Xi_{\alpha}^{\text {g.f. }}(\theta, \beta, \text { dis }),}
\end{gathered}
$$

and we then derive the result. 
Now we divide the family $\theta$ of external contours into two subfamilies:

$\gamma \in \underline{\theta} \Leftrightarrow\{V(\gamma) \cap\{\mathscr{L}\} \neq \emptyset$, or for each $S$ such that $\partial \mathscr{S}=\mathscr{L}$ it is $V(\gamma) \cap\{\mathscr{S}\} \neq \emptyset\}$, $\gamma \in \underline{\underline{\theta}} \Leftrightarrow\{V(\gamma) \cap\{\mathscr{L}\}=\emptyset$, and there exists $\mathscr{S}$ such that: $\partial \mathscr{S}=\mathscr{L}$ and $V(\gamma) \cap\{\mathscr{S}\}=\emptyset\}$.

Proposition 6.1. Let $D(V)$ the set of all families of mutually compatible contours in $V$ and define:

then:

$$
\psi_{\alpha}(\gamma)=\frac{\Xi_{\alpha}^{\text {g.f. }}\left(\gamma, \beta_{t}, \text { dis }\right)}{\Xi_{\alpha}^{\text {g.f. }}\left(\operatorname{Int} \gamma, \beta_{t}\right)},
$$

a)

b)

$$
\begin{gathered}
\Xi_{\alpha}^{\text {g.f. }}\left(V, \beta_{t}\right)=\sum_{\partial \in D(V)} \prod_{\gamma \in \partial} \psi_{\alpha}(\gamma), \\
\left.\psi_{\alpha}(\gamma)\right)=\phi(\gamma) \quad \text { if } \quad \gamma \in \underline{\theta}, \\
\left|\psi_{\alpha}(\gamma)\right| \leqq \phi(\gamma) \quad \text { if } \quad \gamma \in \underline{\theta} .
\end{gathered}
$$

c)

Proof. The proof of statement a) is the same as in Proposition 5.1. Moreover for any $\gamma \in \underline{\theta}$ it is:

$$
\begin{aligned}
& \Xi_{\alpha}^{\text {g.f. }}(\gamma, \beta, \text { dis })=\Xi^{\text {g.f. }}(\gamma, \beta, \text { dis }), \\
& \Xi_{\alpha}^{\text {g.f. }}(\operatorname{Int} \gamma, \beta)=\Xi^{\text {g.f. }}(\operatorname{Int} \gamma, \beta),
\end{aligned}
$$

from which statement b) follows. Since $\Xi^{\text {g.f. }}(\gamma, \beta$, dis $)$ and $\Xi_{\alpha}^{\text {g.f. }}(\operatorname{Int} \gamma, \beta)$ are nonnegative functions, the inequality in statement $c$ ) is a consequence of:

$$
\frac{\mid \Xi_{\alpha}^{\text {g.f. }}(\gamma, \beta, \text { dis }) \mid}{\Xi^{\text {g.f. }}(\gamma, \beta, \text { dis })} \leqq \frac{\Xi_{\alpha}^{\text {g.f. }}(\operatorname{Int} \gamma, \beta)}{\Xi^{\text {g.f. }}(\operatorname{Int} \gamma, \beta)} .
$$

From Lemma 4.1 we deduce that (6.3) is equivalent to:

$$
\frac{\left|\left[\chi_{\gamma ; K} e^{\frac{2 i \pi}{q} \alpha d \sigma(\mathscr{S})}\right]_{K}(\beta)\right|}{\left[\chi_{\gamma ; K}\right]_{K}(\beta)} \leqq \frac{\left[\delta(K \backslash \operatorname{Int} \gamma) e^{\frac{2 i \pi}{q} \alpha d \sigma(\mathscr{S})}\right]_{K}(\beta)}{[\delta(K \backslash \operatorname{Int} \gamma)]_{K}(\beta)},
$$

where $\chi_{\gamma ; K}$ is defined in (5.1). Now we define the following measure:

$$
\langle\cdot\rangle(K,\{J\})=\frac{\sum_{\sigma \in C^{1}(K)} \cdot \prod_{p \in K} e^{J_{p} d \sigma(p)}}{\sum_{\sigma \in C^{1}(K)} \cdot \prod_{p \in K} e^{J_{p} d \sigma(p)}}
$$

in terms of which we rewrite (6.4) as:

$$
\left|\left\langle e^{\frac{2 i \pi}{q} \alpha d \sigma(\mathscr{S})}\right\rangle\left(K,\left\{J^{\prime}\right\}\right)\right| \leqq\left\langle e^{\frac{2 i \pi}{q} \alpha d \sigma(\mathscr{S})}\right\rangle\left(K,\left\{J^{\prime \prime}\right\}\right),
$$

where $\left\{J^{\prime}\right\}$ and $\left\{J^{\prime \prime}\right\}$ satisfy:

$$
\begin{aligned}
& J_{p}^{\prime} \rightarrow \infty \quad \text { if } \quad p \in K \backslash V(\gamma), J_{p}^{\prime \prime} \rightarrow \infty \quad \text { if } \quad p \in K \backslash V(\gamma), \\
& J_{p}^{\prime} \rightarrow-\infty \text { if } p \in \gamma, \\
& J_{p}^{\prime}=\beta \quad \text { if } \quad p \in \operatorname{Int} \gamma, J_{p}^{\prime \prime}=\beta \quad \text { if } p \in \operatorname{In} t \gamma \text {. }
\end{aligned}
$$


The formula (6.5) is a consequence of Ginibre inequality [22], which thus implies statement c).

Now we deduce from the relation (6.1) and Propositions 5.1 and 6.1:

$$
\begin{aligned}
\langle q \delta[\sigma(\mathscr{L})]-1\rangle_{K}^{0}(\beta) & =\sum_{\alpha=1}^{q-1} \frac{\sum_{\partial \in D(K)} \prod_{\gamma \in \partial} \psi_{\alpha}(\gamma)}{\sum_{\partial \in D(K)} \prod_{\gamma \in \partial} \phi(\gamma)} \\
& =\sum_{\alpha=1}^{q-1} e^{\sum^{C} \psi_{\alpha}^{T}(C)-\sum_{C} \phi^{T}(C)},
\end{aligned}
$$

where the sums in the exponent are over all clusters (i.e. families of contours in $D(K)$ satisfying certain conditions). The truncated contour functionals $\phi^{T}$ and $\psi_{\alpha}^{T}$ may (cf. $[23,10,24])$ explicitly be defined by:

$$
\phi^{T}(C)=\sum_{B \subset C}(-1)^{|C|-|B|} \log \sum_{\partial \subset B} \phi(B),
$$

and analogously for $\psi_{\alpha}^{T}$, where $|C|$ denotes the number of contours in $C$. For a $\tau$-functional one has a bound

$$
\sum_{C}^{*}\left|\psi^{T}(C)\right| \leqq k_{1} e^{-\tau}, \quad k_{1}>0,
$$

where $|\cdot|$ denotes the absolute value and the sum is over all clusters such that some of their contour contains a fixed cell. Referring to the explicit formula (6.6) one has $\phi^{T}(C)=\psi_{\alpha}^{T}(C)$ whenever all contours from $C$ are in $\theta$. If it is not the case we say that $C$ is incompatible with $\{\mathscr{L}\}$ and write $C i\{\mathscr{L}\}$. Thus we finally get

$$
\begin{aligned}
& \frac{1}{q-1}\langle q \delta[\sigma(\mathscr{L})]-1\rangle_{K}^{0}(\beta)=\frac{1}{q-1} \sum_{\alpha=1}^{q-1} e^{c: C_{i}\{\mathscr{L}\}}\left[\psi_{\alpha}^{T}(C)-\phi^{T}(C)\right] \\
& \geqq e^{-2 k_{1} e^{-\tau} \operatorname{Per}(\mathscr{L})} .
\end{aligned}
$$

\subsection{Proof of Statement a) of Theorem 2.2: Area Law Decay}

We consider the Wilson parameter with free boundary condition $\langle q \delta[\sigma(\mathscr{L})]-1\rangle_{K}^{f}(\beta)$, where $K$ is the envelope of a rectangular box $K=E(\Lambda) \supset\left\{\mathscr{S}_{0}\right\}$. The duality transformation, taking into account $H_{2}(K)=\{0\}$, leads to:

$$
\langle q \delta[\sigma(\mathscr{L})]-1\rangle_{K}^{f}(\beta)=\sum_{m=1}^{q-1} \frac{\mathbf{Z}_{m}\left(K^{*}, \beta^{*}\right)}{\mathbf{Z}\left(K^{*}, \beta^{*}\right)},
$$

where

$$
\mathbf{Z}_{m}\left(K^{*}, \beta^{*}\right)=\sum_{\sigma \in C^{1}\left(K^{*}\right)} e^{-\beta^{*} H_{K *}(d \sigma+m *(\mathscr{P})} .
$$

$\mathscr{S}$ is a 2 -chain in $K$ such that $\partial \mathscr{S}=\partial \mathscr{S}_{0}=\mathscr{L}$. The 2 -chain $*(\mathscr{S})$ is dual of the 2-chain $\mathscr{S}: *(\mathscr{S})\left(p_{*}\right)=\mathscr{S}(p)$ for any 2-cell $p$ and its dual $p_{*}$.

We shall expand $\mathbf{Z}_{m}\left(K^{*}, \beta^{*}\right)$ in terms of external contours.

Here a plaquette $p_{*}$ is said to be ordered if $(d \sigma+m *(\mathscr{S}))\left(p_{*}\right)=0$, and disordered otherwise. Therefore for every configuration $\sigma \in C^{1}\left(K^{*}\right)$ 
- the 3-cells (cubes) $c_{*} \in\{\mathscr{L}\}^{*}$ are such that plaquettes in their boundaries are not all ordered.

- a 3-cell $c_{*}$ of $K^{*} /\{\mathscr{L}\}^{*}$ cannot have five ordered plaquettes and the sixth disordered. This is a consequence of the identity $d d=0$.

It follows that every configuration $\sigma$ contains a family of mutually external contours $\theta_{*}$ satisfying:

1. $\sigma \in\left\{\sigma \in C^{1}\left(K^{*}\right) /\right.$ for every $p_{*}$ in $\operatorname{Ext}_{K^{*}} \theta_{*}$ it is $(d \sigma+m *(\mathscr{S}))\left(p_{*}\right)=0$, and for every $p_{*}$ in $\theta_{*}$ it is $\left.(d \sigma+m *(\mathscr{S}))\left(p_{*}\right) \neq 0\right\}$.

2. In each such family $\theta_{*}$ there exists a unique contour $\Gamma_{*}$ such that $\{\mathscr{L}\}^{*}$ $\mathrm{C} V\left(\Gamma_{*}\right)$ and for every such contour $\Gamma_{*}$ there exists an integral 2-chain $\mathscr{S}_{\Gamma_{*}}$ such that: $\partial \mathscr{S}_{\Gamma_{*}}=\mathscr{L}^{*}$ and $\left\{\mathscr{I}_{\Gamma_{*}}\right\} \subset V\left(\Gamma_{*}\right) . \mathbf{Z}_{m}\left(K^{*}, \beta^{*}\right)$ is then expanded as:

$$
\mathbf{Z}_{m}\left(K^{*}, \beta^{*}\right)=\sum_{\substack{\Gamma_{*} \\\{\mathscr{L}\}^{*} \subset V\left(\Gamma_{*}\right)}} \sum_{\theta_{*} \supset \Gamma_{*}} \sum_{\sigma \in \mathcal{C}^{1}\left(K^{*}\right)} \chi_{m} e^{-\beta^{*} H_{K^{*}}\left(d \sigma+m \mathscr{S}_{\left.\Gamma_{*}\right)}\right)}
$$

where

$$
\chi_{m}=\prod_{p \in \mathrm{Ext}_{K} \cdot \theta_{*}} \delta[d \sigma(p)] \prod_{p \in \theta_{*}}\left(1-\delta\left[\left(d \sigma+m \mathscr{S}_{\Gamma_{*}}\right)(p)\right]\right) .
$$

From Lemma 4.1 we get:

$$
\mathbf{Z}_{m}\left(K^{*}, \beta^{*}\right)=\sum_{\substack{\Gamma_{*} \\\{\mathscr{L}\}^{*} \subset\left(\Gamma_{*}\right)}} \sum_{* \supset \Gamma_{*}}\left|Z^{1}\left(K^{*}\right)\right| \Xi^{\text {g.f. }}\left(\theta_{*} \backslash \Gamma_{*}, \beta^{*}, \text { dis }\right) \Xi_{m}^{\text {g.f. }}\left(\Gamma_{*}, \beta^{*}, \text { dis }\right),(6.8)
$$

where

$$
\Xi_{m}^{\mathrm{g} . \mathrm{f}}\left(\Gamma_{*}, \beta^{*}, \mathrm{dis}\right)=\sum_{z \in Z^{2}\left(V\left(\Gamma_{*}\right)\right)} e^{-\beta^{*} H_{V\left(\Gamma_{*}\right)}\left(z+m \mathscr{S}_{\Gamma_{*}}\right)} \prod_{p \in \Gamma_{*}}\left(1-\delta\left[\left(z+m \mathscr{S}_{\Gamma_{*}}\right)(p)\right]\right) .
$$

We then prove as in Lemma 4.2:

$$
\Xi_{m}^{\text {g.f. }}\left(\Gamma_{*}, \beta^{*}, \mathrm{dis}\right) \leqq D\left(\gamma_{*}, \beta^{*}\right) \Xi^{\text {g.f. }}\left(\left(\operatorname{Int} \Gamma_{*}\right)^{*}, \beta\right) .
$$

Referring to the proof of Proposition 5.1 we get:

$$
\begin{aligned}
\Xi_{m}^{\text {g.f. }}\left(\Gamma_{*}, \beta_{t}, \text { dis }\right) & \leqq D\left(\Gamma_{*}, \beta_{t}\right) \frac{\Xi^{\text {g.f. }}\left(\left(\operatorname{Int} \Gamma_{*}\right)^{*}, \beta_{t}\right)}{\Xi^{\text {g.f. }}\left(\operatorname{Int} \Gamma_{*}, \beta_{t}\right)} \Xi^{\text {g.f. }}\left(\operatorname{Int} \Gamma_{*}^{\circ}, \beta_{t}\right) \\
& \leqq q^{-k_{0} N^{\circ}\left(\left(I_{*}\right)^{*}\right)} \Xi^{\text {g.f.f. }}\left(\operatorname{Int} \Gamma_{*}, \beta_{t}\right) .
\end{aligned}
$$

On the other hand we have:

$$
\Xi^{\text {g.f. }}\left(\theta_{*} \backslash \Gamma_{*}, \beta_{t}, \operatorname{dis}\right)=\prod_{\gamma_{*}^{\prime} \in \theta_{*} \backslash I_{*}} \phi\left(\gamma_{*}^{\prime}\right) \Xi^{\text {g.f. }}\left(\operatorname{Int}\left(\theta_{*} \backslash \Gamma_{*}\right), \beta_{t}\right) .
$$

Iterating Lemma 5.1 on $\Xi^{\text {g.f. }}$ (Int $\left.\Gamma_{*}, \beta_{t}\right)$ and $\Xi^{\text {g.f. }}\left(\operatorname{Int}\left(\theta_{*} \backslash \Gamma_{*}\right), \beta_{t}\right)$, we deduce:

$$
\begin{aligned}
& \sum_{\theta_{*}: \theta_{*} \supset \Gamma_{*}} \Xi^{\text {g.f. }}\left(\theta_{*} \backslash \Gamma_{*}, \beta^{*}, \text { dis }\right) \Xi_{m}^{\text {g.f. }}\left(\Gamma_{*}, \beta^{*}, \text { dis }\right) \\
& \leqq q^{-k_{0} N^{0}\left(\left(\Gamma_{*}\right)^{*}\right)} \Xi^{\text {g.f. }}\left(\operatorname{Int} \Gamma_{*}, \beta_{t}\right) \sum_{\theta_{*}: \theta_{*} \supset \Gamma_{*}} \prod_{\gamma_{*}^{\prime} \in \theta_{*} \backslash \Gamma_{*}} \phi\left(\gamma_{*}^{\prime}\right) \Xi^{\text {g.f. }}\left(\operatorname{Int}\left(\theta_{*} \backslash \Gamma_{*}\right), \beta_{t}\right)
\end{aligned}
$$

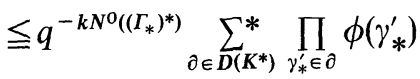

$$
\begin{aligned}
& \leqq q^{-k N^{0}\left(\left(\Gamma_{*}\right)^{*}\right)} \sum_{\partial \in D\left(K^{*}\right)} \prod_{\gamma_{*} \in \partial} \phi\left(\gamma_{*}\right) \\
& =q^{-k N^{0}\left(\left(\Gamma_{*}\right)^{*}\right)} \Xi^{\text {g.f. }}\left(K^{*}, \beta_{t}\right) \text {, }
\end{aligned}
$$


where the $\sum^{*}$ is over all the $\gamma_{*}^{\prime}$ compatible with $\Gamma_{*}$. We use finally that $H^{2}\left(K^{*}\right)$ $=H_{2}(K)=\{0\}$ and (2.4) to get:

$$
\left|Z^{1}\left(K^{*}\right)\right| \Xi^{\text {g.f. }}\left(K^{*}, \beta_{t}\right)=\left|Z^{1}\left(K^{*}\right)\right| \mathbf{Z}^{\text {g.f. }}\left(K^{*}, \beta_{t}\right)=\mathbf{Z}\left(K^{*}, \beta_{t}\right) .
$$

It then follows from (6.7)-(6.10):

$$
\langle q \delta[\sigma(\mathscr{L})]-1\rangle_{K}^{0}\left(\beta_{t}\right) \leqq \sum_{m=1}^{q-1} \sum_{\substack{\Gamma_{*}: \\\{\mathscr{L}\}^{*} \subset V\left(\Gamma_{*}\right)}} q^{-k N^{0}\left(\left(\Gamma_{*}\right)^{*}\right)},
$$

and we deduce the area law decay at the transition point $\beta_{t}$ since the minimal number $N^{0}\left(\left(\Gamma_{*}\right)^{*}\right)$ is at least $N^{2}\left(\mathscr{S}_{0}\right)$. We extend the result for all values of $\beta \leqq \beta_{t}$ by using Ginibre's inequalities [22].

\section{Appendix A: Cell Complex Formalism}

\section{A.1. General Definitions}

The cell complex formalism is very efficient dealing with topological problems inherent to the $q$-states Potts gauge model. We shall first introduce it in an abstract sense along the lines of [13] (cf. also [26, 27]); and then consider its particular example a hypercubical lattice $\mathbb{Z}^{d}$.

A cell complex $K$ is a set whose elements are called cells such that:

- A non-negative integer called dimension is assigned to each cell. The upper bound of the dimensions of all cells is called the dimension of the complex.

- To each cell $s^{p}$ of dimension $p$ (a $p$-cell) corresponds another $p$-cell $\left(-s^{p}\right)$ of the same dimension and called the cell of opposite orientation

- An integer $I\left(s^{p} ; s^{p-1}\right)$ called the incidence number is assigned to all pairs of cells $\left(s^{p}, s^{p-1}\right)$ in a such way that

$$
I\left(-s^{p} ; s^{p-1}\right)=I\left(s^{p} ;-s^{p-1}\right)=-I\left(s^{p} ; s^{p-1}\right) .
$$

A cell complex is called an a-complex if

$$
\sum_{s^{p-1}} I\left(s^{p} ; s^{p-1}\right) I\left(s^{p-1} ; s^{p-2}\right)=0
$$

for any two cells $s^{p}$ and $s^{p-2}$ belonging to $K$. Another terminology is sometimes used: a cell space instead of a cell complex and a cell complex instead of an $a$-complex; the above have been introduced in [13].

An integral p-chain $c^{p}$ on the complex $K$ is an odd function on $p$-cells with values in $\mathbb{Z}$, the group of integers.

$$
c^{p}: s^{p} \in K \rightarrow c^{p}\left(s^{p}\right) \in \mathbb{Z} .
$$

The set of all $p$-chains over $K$ form an abelian group denoted by $C^{p}(K)$. The rank of this group is denoted $N^{p}(K) ; 2 N^{p}(K)$ is the number of $p$-cells of $K$.

A monomial chain $m \cdot s^{p}$ is a chain that takes a value $m$ on $s^{p}$ and vanishes on all $p$-cells different than $s^{p}$. Hence any integral chain may be written as a sum of 
monomial chains:

$$
c^{p}=\sum_{i} m_{i} \cdot s_{i}^{p}, \quad 1 \leqq i \leqq N^{p}(K), m_{i}=c^{p}\left(s_{i}^{p}\right) .
$$

Hereafter $s^{p}$ denotes either the cell $s^{p}$ or the monomial chain $1 \cdot s^{p}$.

On $C^{p}(K)$ one may introduce the scalar product,

The operator

$$
\left(c^{p}, c^{\prime p}\right)=\sum_{i} m_{i} \cdot m_{i}^{\prime}
$$

$$
\begin{aligned}
& \partial s^{p}=\sum_{j} I\left(s^{p} ; s_{j}^{p-1}\right) s_{j}^{p-1}, \\
& \partial c^{p}=\sum_{i} m_{i} \cdot \partial s_{i}^{p}=\sum_{i ; j} m_{i} I\left(s_{i}^{p} ; s_{j}^{p-1}\right) s_{j}^{p-1}
\end{aligned}
$$

defines a homomorphism of the group $C^{p}(K)$ into the group $C^{p-1}(K)$ and is called the boundary operator. The dual coboundary operator $\partial^{*}$ (the adjoint of $\partial$ with respect to the scalar product) defines a homomorphism of the group $C^{p}(K)$ into the group $C^{p+1}(K)$ :

$$
\left(\partial c^{p}, c^{p-1}\right)=\left(c^{p}, \partial^{*} c^{p-1}\right)
$$

In particular:

$$
\begin{aligned}
& \partial^{*} s^{p}=\sum_{j} I\left(s_{j}^{p+1}, s^{p}\right) s_{j}^{p+1}, \quad \text { and } \\
& \partial^{*} c^{p}=\sum_{i} m_{i} \cdot \partial s_{i}^{p}=\sum_{i ; j} m_{i} I\left(s_{j}^{p+1}, s_{i}^{p}\right) s_{j}^{p+1} .
\end{aligned}
$$

Notice that

$$
I\left(s^{p} ; s^{p-1}\right)=\left(\partial s^{p}, s^{p-1}\right)=\left(s^{p}, \partial^{*} s^{p-1}\right) .
$$

A cell complex $K_{0}$ is said to be a cell subcomplex of the complex $K$ if every element of $K_{0}$ is an element of $K$, every two cells $s^{p}$ and $s^{p+1}$ have the same incidence number in $K$ as they do in $K_{0}$ and every pair of opposites in $K_{0}$ is a pair of opposites in $K$. A cell complex $K_{0}$ is said to be closed (respectively open) if it contains with every cell also the cells on its boundary (respectively coboundary). We denote by $\bar{K}_{0}$ the closure of $K_{0}$, i.e. the minimal closed cell-complex containing $K_{0}$. A complex is said to be connected if it cannot be expressed as the union of two non-empty disjoint closed subcomplexes.

A hypercubic lattice $\mathbb{Z}^{d}$ may be considered as a cell complex denoted $\mathbb{L}$. Its 0 -cells are vertices, its 1-cells are bonds, its 2-cells are plaquettes etc. We shall denote $\mathbb{L}^{p}, p=0,1, \ldots, d$, the set of $p$-cell in $\mathbb{L}$. The orientation is the usual one and the incidence number $I\left(s^{p} ; s^{p-1}\right)$ takes values \pm 1 if $s^{p-1}$ belongs to the boundary of $s^{p}$ with respect to the relative orientation and the value 0 otherwise.

Let us consider a cell subcomplex $K$ of $\mathbb{L}$ and restrict the incidence function to $K$ (note that the boundary operation, then, does not coincide with the same operation in IL). $K$ will be an $a$-complex if it satisfies (A.1). In particular closed and open subcomplexes are $a$-complexes. Hereafter we shall only consider $a$-complexes. 
The group $C^{p}(K)$ has two distinguished subgroups with respect to the operator $\partial$ : the group of $p$-cycles $Z_{p}(K)=\left\{c^{p} \mid \partial c^{p}=0\right\}$ and the group of $p$-boundaries $B_{p}(K)$ $=\left\{c^{p} \mid c^{p}=\partial c^{p+1}\right\}$. Since $\partial \partial=0$ as follows from (A.1), every boundary is a cycle: $B_{p}(K) \subset Z_{p}(K)$. The converse is not true in general. Consider for example the 2-dimensional complex shown in Fig. A.1, with an omitted plaquette. We have $\partial c_{1}=0=\partial c_{2}$, but neither $c_{1}$ or $c_{2}$ is a boundary. However $c_{2}=c_{1}+\partial s$, where $s$ is a two-chain sum of the plaquette spanned between $c_{1}$ and $c_{2}$. Two such 1-cycles are called homologous; two cycles are called homologous if they differ by a boundary. The factor group $H_{p}(K) \equiv Z_{p}(K) / B_{p}(K)$, whose elements are the equivalent classes of homologous $p$-cycles, is called the $p$-homology group of the complex $K$. The rank of $H_{p}(K)$ denoted by $\pi^{p}(K)$ is a topological invariant called the $p^{\text {th }}$-Betti number that characterizes the number of independent $p$-dimensional holes in $K$.

For $\partial^{*}$ one defines similarly the groups of $p$-cocycles $Z^{p}(K)$ and $p$-coboundaries $B^{p}(K)$ and the $p$-cohomology group $H^{p}(K)$ of a complex $K$.

The others topological invariants are the $p$-torsion coefficients, $\theta_{i}^{p}$ (see Appendix A.3): they correspond to the fact that it may exist $p$-chains $c^{p}$ which are not boundaries of $(p+1)$-chains in the considered complex, while $\theta_{i}^{p} c^{p}$ are boundaries. The number $\tau^{p}$ of $p$-torsion coefficients is also a topological invariant called $p$-torsion number. A characteristic example is the Klein Bottle shown in Fig. A.2: the 1-cycle $2 \times(04+43+30)$ (with obvious notations) is the boundary of the 2-chain sum of the plaquette oriented clockwise whereas the 1-cycle $04+43+30$ is not a boundary; $\tau^{1}=1$ and $\theta^{1}=2$.

The following notations serve to describe configurations of lattice models. A homomorphism $\sigma^{p}$ from $C^{p}(K)$ into an abelian group $G$ is called a $G$-valued $p$-cochain. The set of $G$-valued $p$-cochains of a complex $K$ forms an abelian group denoted $C^{p}(K, G)$, in particular $C^{p}(K, \mathbb{Z})=C^{p}(K)$, and the scalar product can be considered as the product of an integral chain with an integral cochain. Any $\sigma^{p}$ is determined by its values on the chains $1 \cdot s^{p}$, i.e. on the cells $s^{p}$; it thus defines an odd function on the complex $K$ with values in $G$.

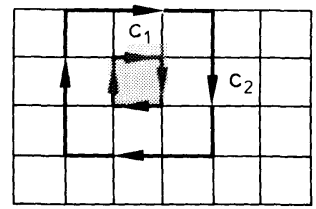

Fig. A.1. Homologous non-bounding cycles

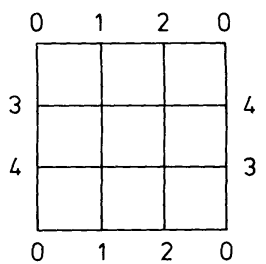

Fig. A.2. A Klein bottle: such a complex may be obtained as a subcomplex of a $d$-dimensional lattice when $d \geqq 4$ (obviously it needs more cells than these shown) 
One may define the differential

$$
d: C^{p}(K, G) \rightarrow C^{p+1}(K, G)
$$

and the codifferential

$$
d^{*}: C^{p}(K, G) \rightarrow C^{p-1}(K, G)
$$

operators:

$$
d \sigma^{p}\left(c^{p+1}\right)=\sigma^{p}\left(\partial c^{p+1}\right) \text { and } d^{*} \sigma^{p}\left(c^{p-1}\right)=\sigma^{p}\left(\partial^{*} c^{p-1}\right) .
$$

In particular

$$
\begin{aligned}
d \sigma^{p}\left(s^{p+1}\right) & =\sum_{j} I\left(s_{j}^{p+1} ; s^{p}\right) \sigma^{p}\left(s_{j}^{p}\right), \\
d^{*} \sigma^{p}\left(s^{p-1}\right) & =\sum_{j} I\left(s_{j}^{p} ; s^{p-1}\right) \sigma^{p}\left(s_{j}^{p}\right) .
\end{aligned}
$$

Whenever $G$ is a ring with unity every $G$-valued $p$-cochain $\sigma$ belonging to $C^{p}(K, G)$ has a unique decomposition on the cell basis: $\sigma=\sum_{i} \alpha_{i} s_{i}^{p}$, here $\alpha_{i}$ belongs to $G$ and $\sum$ denotes the group law of $G$.

We introduce

- the group of $G$-valued $p$-cycles of $K Z_{p}(K, G)=\left\{\sigma^{p} \in C^{p}(K, G) \mid d^{*} \sigma^{p}=0\right\}$ (here 0 denotes the unit element of $G$ )

- the group of $G$-valued $p$-boundaries of $K$

$$
B_{p}(K, G)=\left\{\sigma^{p} \in C^{p}(K, G) \mid \sigma^{p}=d^{*} \sigma^{p+1}, \sigma^{p+1} \in C^{p+1}(K, G)\right\},
$$

the group of $G$-valued $p$-cocycles of $K Z^{p}(K, G)=\left\{\sigma^{p} \in C^{p}(K, G) \mid d \sigma^{p}=0\right\}$

- the group of $G$-valued $p$-coboundaries of $K$

$$
B^{p}(K, G)=\left\{\sigma^{p} \in C^{p}(K, G) \mid \sigma^{p}=d \sigma^{p-1}, \sigma^{p-1} \in C^{p-1}(K, G)\right\} .
$$

The factor groups $H^{p}(K, G)=Z p(K, G) / B p(K, G), H^{p}(K, G)=Z^{p}(K, G) / B^{p}(K, G)$ are respectively the $G$-valued $p$-homology and the $G$-valued $p$-cohomology groups of $K$.

\section{A.2. Dual Lattice and Dual Complex}

Let $K$ be a $d$-dimensional cell complex, $K^{*}$ is said to be the dual complex of $K$ if there is a one-to-one correspondence

$$
s^{p} \rightarrow *\left(s^{p}\right)=s_{*}^{d-p}
$$

between the $p$-cells, $s^{p}$, of $K$ and the $(d-p)$-cells, $s_{*}^{d-p}$, of $K^{*}$ such that the incidence numbers satisfy the relation

$$
I\left(s^{p} ; s^{p-1}\right)=I\left(s_{*}^{d-p+1} ; s_{*}^{d-p}\right) .
$$

The lattice $\left(\mathbb{Z}^{d}\right)^{*}=\left\{x_{*} / x_{*}=\left(x^{1}+\frac{1}{2}, \ldots, x^{i}+\frac{1}{2}, \ldots, x^{d}+\frac{1}{2}\right), x^{i} \in \mathbb{Z}\right\}$ is the dual lattice of $\mathbb{Z}^{d}$. Let $\mathbb{L}^{*}$ be the complex associated with $\left(\mathbb{Z}^{d}\right)^{*} . \mathbb{L}^{*}$ is the dual complex of $\mathbb{L}$. For any cell subcomplex $K$ of $\mathbb{L}$ there is a dual complex $K^{*}$ which is a subcomplex of $\mathbb{L}^{*}$; if $K$ is closed, $K^{*}$ is open, if $K$ is open $K^{*}$ is closed. In this article we consider 
a 4-dimensional lattice cell complex; the dual of a site is an hypercube (and conversely), the dual of a link is a cube (and conversely), and the dual of a plaquette is a plaquette.

We introduce the operation $*\left(\right.$ Hodge operation) mapping $C^{p}(K)$ into $C^{d-p}\left(K^{*}\right)$ and $C^{p}(K, G)$ into $C^{d-p}\left(K^{*}, G\right)$ by:

$$
\begin{aligned}
*: c^{p} \rightarrow *\left(c^{p}\right)=c_{*}^{d-p}, & c_{*}^{d-p}\left(s_{*}^{d-p}\right)=c^{p}\left(s^{p}\right), \\
*: \sigma^{p} \rightarrow *\left(\sigma^{p}\right)=\sigma_{*}^{d-p}, & \sigma_{*}^{d-p}\left(s_{*}^{d-p}\right)=\sigma^{p}\left(s^{p}\right) .
\end{aligned}
$$

It follows from (A.3) and (A.5) that

$$
*\left(d \sigma^{p}\right)=d^{*} *\left(\sigma^{p}\right), \quad *\left(d^{*} \sigma^{p}\right)=d *\left(\sigma^{p}\right) ;
$$

and for integral chains

$$
*\left(\partial c^{p}\right)=\partial^{*} *\left(c^{p}\right), \quad *\left(\partial^{*} c^{p}\right)=\partial *\left(c^{p}\right) .
$$

Therefore the mapping $*$ determines an isomorphism between

1. The group of $G$-valued $p$-cycles of $K, Z_{p}(K, G)$, and the group of $G$-valued $d$-p-cocycles of $K^{*}, Z^{d-p}\left(K^{*}, G\right)$,

2. The group of $G$-valued $p$-boundaries of $K, B_{p}(K, G)$, and the group of $G$-valued $(d-p)$-coboundaries of $K^{*}, B^{d-p}\left(K^{*}, G\right)$,

3. The $G$-valued $p$-homology group of $K, H_{p}(K, G)$, and the $G$-valued $(d-p)$ cohomology group of $K^{*}, H^{d-p}\left(K^{*}, G\right)$.

\section{A.3. $\partial$-Basis and $\partial^{*}$-Basis}

A standard result of algebraic topology is that the group $C^{p}(K)$ admits a canonical $\partial$-basis and $\partial^{*}$-basis. A $\partial$-basis consists in five families of integral $p$-chains:

$$
\begin{gathered}
\left\{a_{i}^{p} \mid i=1 \ldots v^{p-1}\right\}, \quad\left\{x_{i}^{p} \mid i=1 \ldots \tau^{p-1}\right\}, \quad\left\{h_{i}^{p} \mid i=1 \ldots \pi^{p}\right\}, \\
\left\{b_{i}^{p} \mid i=1 \ldots v^{p}\right\}, \quad \text { and } \quad\left\{t_{i}^{p} \mid i=1 \ldots \tau^{p}\right\}
\end{gathered}
$$

satisfying,

$$
\begin{array}{ll}
N^{p}=v^{p-1}+\tau^{p-1}+\pi^{p}+v^{p}+\tau^{p} & \\
\partial a_{i}^{p}=b_{i}^{p-1} & i=1 \ldots v^{p-1} \\
\partial x_{i}^{p}=\theta_{i}^{p-1} t_{i}^{p-1} & i=1 \ldots \tau^{p-1} \\
\partial h_{i}^{p}=0 & i=1 \ldots \pi^{p} \\
b_{i}^{p}=\partial a_{i}^{p+1} & i=1 \ldots v^{p} \\
\theta_{i}^{p} t_{i}^{p}=\partial x_{i}^{p+1} & i=1 \ldots \tau^{p} \\
\theta_{i}^{p}=0\left(\bmod \theta_{i+1}^{p}\right) . &
\end{array}
$$

$\theta_{i}^{p}$ is the order of the element $t_{i}^{p}$ relative to the group $B_{p}(K)$ (see [13]). A $\partial^{*}$-basis consists in five families of integral $p$-chains:

$$
\begin{gathered}
\left\{\bar{a}_{i}^{p} \mid i=1 \ldots v^{p}\right\}, \quad\left\{\bar{x}_{i}^{p} \mid i=1 \ldots \tau^{p}\right\}, \quad\left\{\bar{h}_{i}^{p} \mid i=1 \ldots \pi^{p}\right\}, \\
\left\{\bar{b}_{i}^{p} \mid i=1 \ldots v^{p-1}\right\}, \quad \text { and } \quad\left\{\bar{t}_{i}^{p} \mid i=1 \ldots \tau^{p-1}\right\}
\end{gathered}
$$


satisfying,

$$
\begin{array}{ll}
N^{p}=v^{p-1}+\tau^{p-1}+\pi^{p}+v^{p}+\tau^{p} & \\
\partial^{*} \bar{a}_{i}^{p}=\bar{b}_{i}^{p+1} & i=1 \ldots v^{p} \\
\partial^{*} \bar{x}_{i}^{p}=\theta_{i}^{p-1} \bar{t}_{i}^{p+1} & i=1 \ldots \tau^{p} \\
\partial^{*} \bar{h}_{i}^{p}=0 & i=1 \ldots \pi^{p} \\
\bar{b}_{i}^{p}=\partial^{*} \bar{a}_{i}^{p-1} & i=1 \ldots v^{p-1} \\
\theta_{i}^{p} \bar{t}_{i}^{p}=\partial^{*} \bar{x}_{i}^{p-1} & i=1 \ldots \tau^{p-1} .
\end{array}
$$

We refer to [13] and [27] for suitable examples for our purposes.

The mapping (A.4) sends a $\partial$-basis in a $\partial^{*}$-basis and conversely, and

$$
\begin{aligned}
& N^{p}(K)=N^{d-p}\left(K^{*}\right), \quad \pi^{p}(K)=\pi^{d-p}\left(K^{*}\right) \quad 0 \leqq p \leqq d, \\
& \tau^{p-1}(K)=\tau^{d-p}\left(K^{*}\right), \quad 1 \leqq p \leqq d .
\end{aligned}
$$

Every $p$-cycle, $z$, belonging to the group $Z_{p}(K, G)$ has a unique decomposition on a $\partial$-basis

$$
z=\sum_{i=1}^{\tau^{p-1}} \xi_{i} x_{i}^{p}+\sum_{i=1}^{\pi^{p}} \mu_{i} h_{i}^{p}+\sum_{i=1}^{v^{p}} \beta_{i} b_{i}^{p}+\sum_{i=1}^{\tau^{p}} \gamma_{i} t_{i}^{p} .
$$

Here $\mu_{i}, \beta_{i}, \gamma_{i}$ belong to $G$ and $\xi_{i}$ belongs to the group $G\left(\theta_{i}^{p-1}\right)=\left\{g \in G / \theta_{i}^{p-1} g=0\right\}$. Every $p$-boundary, $b$, belonging to the group $B_{p}(K, G)$ has a unique decomposition on a $\partial$-basis:

$$
b=\sum_{i=1}^{\nu p} \beta_{i} b_{i}^{p}+\sum_{i=1}^{\tau^{p}} \varrho_{i} t_{i}^{p}
$$

Here $\beta_{i}$ belong to $G$ and $\varrho_{i}$ belongs to the group $\theta_{i}^{p} G=\left\{\theta_{i}^{p} g / g \in G\right\}$. Every $p$-cocycle, $z^{\prime} \in Z^{p}(K, G)$ has a unique decomposition on a $\partial^{*}$-basis:

$$
z^{\prime}=\sum_{i=1}^{\tau^{p}} \xi_{i}^{\prime} \bar{x}_{i}^{p}+\sum_{i=1}^{\pi^{p}} \mu_{i}^{\prime} \bar{h}_{i}^{p}+\sum_{i=1}^{\nu p-1} \beta_{i}^{\prime} \bar{b}_{i}^{p}+\sum_{i=1}^{\tau^{p-1}} \gamma_{i}^{\prime} \bar{t}_{i}^{p}
$$

Here $\mu_{i}^{\prime}, \beta_{i}^{\prime}, \gamma_{i}^{\prime}$ belong to $G$ and $\xi_{i}^{\prime}$ belongs to the group $G\left(\theta_{i}^{p}\right)$. Every $p$-coboundary, $b^{\prime} \in B_{p}(K, G)$ has a unique decomposition on a $\partial^{*}$-basis:

$$
b^{\prime}=\sum_{i=1}^{\nu p-1} \beta_{i}^{\prime} \bar{b}_{i}^{p}+\sum_{i=1}^{\tau^{p-1}} \gamma_{i}^{\prime} \bar{t}_{i}^{p}
$$

Here $\beta_{i}^{\prime}$ belong to $G$ and $\gamma_{i}^{\prime}$ belongs to the group $\theta_{i}^{p-1} G$. 
For the above groups the following decomposition in direct sums holds true (cf. [13] for details):

$$
\begin{aligned}
& Z_{p}(K, G) \cong \sum_{i=1}^{\tau^{p-1}} G\left(\theta_{i}^{p-1}\right) \oplus \sum_{i=1}^{\pi^{p}} G \oplus \sum_{i=1}^{\nu^{p}} G \oplus \sum_{i=1}^{\tau^{p}} G, \\
& B_{p}(K, G) \cong \sum_{i=1}^{v^{p}} G \oplus \sum_{i=1}^{\tau^{p}} \theta_{i}^{p} G, \\
& H_{p}(K, G) \cong \sum_{i=1}^{\tau^{p-1}} G\left(\theta_{i}^{p-1}\right) \oplus \sum_{i=1}^{\pi^{p}} G \oplus \sum_{i=1}^{\tau^{p}} G / \theta_{i}^{p} G, \\
& Z^{p}(K, G) \cong \sum_{i=1}^{\tau^{p-1}} G \oplus \sum_{i=1}^{v^{p-1}} G \oplus \sum_{i=1}^{\pi^{p}} G \oplus \sum_{i=1}^{\tau^{p}} G\left(\theta_{i}^{p}\right), \\
& B^{p}(K, G) \cong \sum_{i=1}^{\tau^{p-1}} \theta_{i}^{p-1} G \oplus \sum_{i=1}^{\nu^{p-1}} G, \\
& H^{p}(K, G) \cong \sum_{i=1}^{\tau^{p-1}} G / \theta_{i}^{p-1} G \oplus \sum_{i=1}^{\pi^{p}} G \oplus \sum_{i=1}^{\tau^{p}} G\left(\theta_{i}^{p}\right), \\
& N^{p}=v^{p-1}+\tau^{p-1}+\pi^{p}+v^{p}+\tau^{p} .
\end{aligned}
$$

where hereafter the symbols $\cong$ and $\oplus$ denotes respectively isomorphism and direct sum of groups. Whenever $G=\mathbb{Z}_{q}$ one has for any $\theta>1$ (cf. [13] t.2, p. 113):

$$
\mathbb{Z}_{q}(\theta)=\mathbb{Z}_{q} / \theta \mathbb{Z}_{q}=\mathbb{Z}_{(q, \theta)},
$$

where $(q, \theta)$ is the greatest common divisor of $q$ and $\theta$. Therefore

$$
H_{p}\left(K, \mathbb{Z}_{q}\right)=H^{p}\left(K, \mathbb{Z}_{q}\right)=\cong \sum_{i=1}^{\tau^{p-1}} \mathbb{Z}_{q}\left(\theta_{i}^{p-1}\right) \oplus \sum_{i=1}^{\pi^{p}} \mathbb{Z}_{q} \oplus \sum_{i=1}^{\tau^{p}} \mathbb{Z}_{q}\left(\theta_{i}^{p}\right)
$$

We finally recall the Alexander's duality theorem; we refer the reader to [13] (Vol. 3, pp. 41-42).

Theorem A.1 Let $K$ a closed subcomplex of the lattice cell complex $\mathbb{L}$ then:

a) For $p$ such that $1 \leqq p \leqq d-2$ it is

$$
\begin{aligned}
H^{p}(K, G) & \cong H_{d-p-1}\left([\mathbb{L} \backslash K]^{*}, G\right), \text { and } \\
\pi^{p}(K) & =\pi^{d-p-1}\left([\mathbb{L} \backslash K]^{*}\right), \\
\pi^{0}\left([\mathbb{L} \backslash K]^{*}\right)-1 & =\pi^{d-1}(K) .
\end{aligned}
$$

b) For $0 \leqq p \leqq d-2$ it is

$$
\tau^{p}(K)=\tau^{d-p-2}\left([\mathbb{L} \backslash K]^{*}\right) .
$$

c) $K$ and $[\mathbb{L} \backslash K]^{*}$ are $p$-torsion free for $p=0$ and $p \geqq d-2$.

\section{Appendix B: Proof of Lemma 4.3}

We first rewrite the left-hand side of the inequality of the lemma in term of quantities relative to the complex $V(\gamma)^{*}$ dual of the complex $V(\gamma)$. 
Lemma B.1.

$$
\begin{aligned}
& \mid H^{2}\left(V(\gamma) \mid q^{-N^{0}(V(\gamma))+N^{1}(V(\gamma))-\frac{N^{2}(V(\gamma))}{2}}\right. \\
& \quad=\left|H_{1}\left(V(\gamma)^{*}\right)\right| q^{N^{0}\left(V(\gamma)^{*}\right)-N^{1}\left(V(\gamma)^{*}\right)+\frac{N^{2}\left(V(\gamma)^{*}\right)}{2}-1} .
\end{aligned}
$$

Proof. ${ }^{2}$ From the Euler Poincaré formula

$$
\sum_{p}(-1)^{p} N^{p}=\sum_{p}(-1)^{p} \pi^{p}
$$

and (A.7) we get

$$
\begin{aligned}
& -N^{0}(V(\gamma))+N^{1}(V(\gamma))-\frac{N^{2}(V(\gamma))}{2}=N^{0}\left(V(\gamma)^{*}\right)-N^{1}\left(V(\gamma)^{*}\right)+\frac{N^{2}\left(V(\gamma)^{*}\right)}{2} \\
& -\pi^{0}\left(V(\gamma)^{*}\right)+\pi^{1}\left(V(\gamma)^{*}\right)-\pi^{2}\left(V(\gamma)^{*}\right)+\pi^{3}\left(V(\gamma)^{*}\right)-\pi^{4}\left(V(\gamma)^{*}\right) .
\end{aligned}
$$

Since closed complexes are 0 and 2-torsion free (by Theorem A.1) we deduce from (A.9):

$$
q^{-\pi^{1}\left(V(\gamma)^{*}\right)}\left|H_{1}\left(V(\gamma)^{*}\right)\right|=q^{-\pi^{2}\left(V(\gamma)^{*}\right)}\left|H_{2}\left(V(\gamma)^{*}\right)\right|=q^{-\pi^{2}\left(V(\gamma)^{*}\right)}\left|H^{2}(V(\gamma))\right|
$$

We use finally that $\pi^{4}\left(V(\gamma)^{*}\right)=0$ since $V(\gamma)^{*}$ is closed, $\pi^{0}\left(V(\gamma)^{*}\right)=1$ by construction and that $\pi^{3}\left(V(\gamma)^{*}\right)=\pi^{0}(\mathbb{L} \backslash V(\gamma))-1$ (see Theorem A.1) is therefore zero since $\mathbb{L} \backslash V(\gamma) \equiv \operatorname{Ext} \gamma$ has one connected component.

First step: We shall estimate the term

$$
N^{0}\left(V(\gamma)^{*}\right)-N^{1}\left(V(\gamma)^{*}\right)+\frac{N^{2}\left(V(\gamma)^{*}\right)}{2}
$$

For every site $x$ of the dual lattice $\mathbb{L}^{*}$ we define the incidence number $I^{\ell}\left(x, V(\gamma)^{*}\right)$ as the number of links of $V(\gamma)^{*}$ (positively oriented) ${ }^{3}$ which contains $x$, and the incidence number $I^{h}\left(x, V(\gamma)^{*}\right)$ as the number of plaquettes of $V(\gamma)^{*}$ (positively oriented) which contains $x$.

Lemma B.2. For any contour $\gamma$ :

$$
N^{0}\left(V(\gamma)^{*}\right)-N^{1}\left(V(\gamma)^{*}\right)+\frac{N^{2}\left(V(\gamma)^{*}\right)}{2}=-\frac{1}{8} \sum_{x \in V(\gamma)^{*}}\left[4 I^{\ell}\left(x, V(\gamma)^{*}\right)-I^{h}\left(x, V(\gamma)^{*}\right)-8\right] .
$$

\footnotetext{
${ }^{2}$ In this appendix we use the same notations as in Sects. 1-6 and omit the specification of the group $\mathbb{Z}_{q}$ (see Footnote 1)

${ }^{3}$ In this appendix we shall only consider positively oriented cells without specifying this in the following. Moreover we shall say that:

a) a cell $s^{p}$ contains in its boundary a cell $s^{\prime}$ of lower dimension, if the minimal closed subcomplex of $\mathbb{L}$ containing as $p$-cells only the two $p$-cells $s^{p}$ and $\left(-s^{p}\right)$ contains also the cell $s^{\prime}$.

b) a cell $s^{p}$ contains in its coboundary a cell $s^{\prime}$ of greater dimension, if the minimal open subcomplex of $\mathbb{L}$ containing as $p$-cells only the two $p$-cells $s^{p}$ and $\left(-s^{p}\right)$ contains also the cell $s^{\prime}$
} 
Proof. The complex $V(\gamma)^{*}$ is closed, hence every link in $V(\gamma)^{*}$ contains two sites of $V(\gamma)^{*}$ and every plaquette in $V(\gamma)^{*}$ contains four sites of $V(\gamma)^{*}$. Therefore we have

$$
N^{0}\left(V(\gamma)^{*}\right)-N^{1}\left(V(\gamma)^{*}\right)+\frac{N^{2}\left(V(\gamma)^{*}\right)}{2}=\sum_{x \in V(\gamma)^{*}}\left[1-\frac{I^{\ell}\left(x, V(\gamma)^{*}\right)}{2}+\frac{I^{h}\left(x, V(\gamma)^{*}\right)}{8}\right],
$$

from which the lemma follows.

We shall now show that (B.1) is a "boundary term."

\section{Lemma B.3.}

$$
\sum_{x \in V(\gamma)^{*}}\left[4 I^{\ell}\left(x, V(\gamma)^{*}\right)-I^{h}\left(x, V(\gamma)^{*}\right)-8\right] \geqq N^{0}\left(\gamma^{*}\right) .
$$

Proof. A site in the lattice may contain at most 8 links and 24 plaquettes in its coboundary. Since $(\operatorname{Int} \gamma)^{*}$ is open, the sites $x$ of $V(\gamma)^{*}$ such that $I^{\ell}\left(x, V(\gamma)^{*}\right)=8$ and $I^{\prime \prime}\left(x, V(\gamma)^{*}\right)=24$ belong to $(\operatorname{Int} \gamma)^{*}$; these sites will be called saturated sites. The nonsaturated sites of $V(\gamma)^{*}$ belong to $\gamma^{*}$. We observe that the term $\left[4 I^{\ell}\left(x, V(\gamma)^{*}\right)\right.$ $\left.-I^{n}\left(x, V(\gamma)^{*}\right)-8\right]$ gives no contribution for any saturated site. We shall show that this term gives at least a contribution +1 for each non-saturated site, i.e. for each site in $\gamma^{*}$. Clearly this quantity can be negative if we replace $V(\gamma)^{*}$ by a whatever complex, the positivity will follow from the fact that $\gamma$ is a contour. In particular $V(\gamma)^{*}$ is closed and thus all the links in the boundary of a plaquette in $V(\gamma)^{*}$ belongs to $V(\gamma)^{*}$, furthermore we have other constraints which we give in the following

Remark B.1. For any configuration $\sigma$, a cube in $\mathbb{L}$ cannot contain five ordered plaquettes and a disordered one: this follows from $d d=0$. It follows that a link in $V(\gamma)^{*}$ has in its coboundary at least two plaquettes of $V(\gamma)^{*}$.

A first consequence of Remark B.1 is that $I^{\ell}\left(x, V(\gamma)^{*}\right)$ cannot take the value 1 and 2. Let $\mathscr{S}_{m}\left(V(\gamma)^{*}\right)=\left\{x \in V(\gamma)^{*} / I^{\ell}\left(x, V(\gamma)^{*}\right)=m\right\}$, then

$$
\begin{aligned}
\sum_{x \in V(\gamma)^{*}}\left[4 I^{\ell}\left(x, V(\gamma)^{*}\right)-I^{h}\left(x, V(\gamma)^{*}\right)-8\right] & =\sum_{m=3}^{8} \sum_{\substack{x \in V(\gamma)^{*} \\
I^{\ell}\left(x, V(\gamma)^{*}\right)=m}}\left(4 m-I^{h}\left(x, V(\gamma)^{*}\right)-8\right) \\
& =\sum_{m=3}^{8} \sum_{x \in \mathscr{S}_{m}\left(V(\gamma)^{*}\right)}\left(4 m-I^{h}\left(x, V(\gamma)^{*}\right)-8\right) .
\end{aligned}
$$

We now examine the values that can take $I^{h}\left(x, V(\gamma)^{*}\right)$ whenever $I^{\ell}\left(x, V(\gamma)^{*}\right)$ is given, more precisely when the links of $V(\gamma)^{*}$ incident to $x$ are given. We denote $\left\{e_{1}, \ldots, e_{8}\right\}$ the eight unit vectors of $\mathbb{R}^{4}$ in the direction of the axis, and for $3 \leqq m \leqq 8$ let $\left\{e_{i_{1}}, \ldots, e_{i_{m}}\right\}$ be $m$ different elements of $\left\{e_{1}, \ldots, e_{8}\right\}$. For any contour $\gamma$ and for any $x$ in $V(\gamma)^{*}$ we denote

$\left[\gamma^{*}, x\right]\left(e_{i_{1}}, \ldots, e_{i_{m}}\right)=\left\{\left[\gamma^{*}, x\right] / \begin{array}{l}\text { the links }\left(x, x+e_{i_{k}}\right)(k=1, \ldots, m) \text { belongs to } V(\gamma)^{*} \\ \text { the others links }\left(x, x+e_{j}\right) \text { belongs to }(\operatorname{Ext} \gamma)^{*}\end{array}\right\}$.

Given $\left(e_{i_{1}}, \ldots, e_{i_{m}}\right)$ and a couple $\left[\gamma^{*}, x\right]$ in $\left[\gamma^{*}, x\right]\left(e_{i_{1}}, \ldots, e_{i_{m}}\right)$ we look at the possible values of $I^{\prime \prime}\left(x, V(\gamma)^{*}\right)$. For example if $m=4$ and $e_{i_{1}}, \ldots, e_{i_{4}}$ belongs to the same plane, the plaquettes in the coboundary of $x$ which belongs to $V(\gamma) *$ are these of this plane and only these ones (Fig. B.1). Two different values for $I^{h}\left(x, V(\gamma)^{*}\right)$ when four vectors $e_{i_{1}}, \ldots, e_{i_{4}}$ are not in the same plane are also shown in Fig. B.1. 

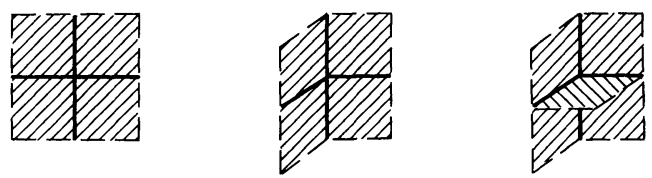

Fig. B.1

We denote

$$
I_{\max }^{h}\left(e_{i_{1}}, \ldots, e_{i_{m}}\right)=\max _{\left(\gamma^{*}, x\right) \in\left(\gamma^{*}, x\right)\left(e_{i_{1}}, \ldots, e_{i_{m}}\right)} I^{h}\left(x, V(\gamma)^{*}\right) .
$$

For a given $m$ we observe that $I_{\max }^{h}\left(e_{i_{1}}, \ldots, e_{i_{m}}\right)$ takes its maximum value when the $m$ vectors $e_{i_{1}}, \ldots, e_{i_{m}}$ are chosen such that the number of orthogonal vectors in this family is maximal. We let

$$
I_{\max }^{h}(m)=\max _{\left(e_{i_{1}}, \ldots, e_{i_{m}}\right)} I_{\max }^{h}\left(e_{i_{1}}, \ldots, e_{i_{m}}\right) .
$$

Remark B.2. If $\left(\gamma^{*}, x\right) \in\left(\gamma^{*}, x\right)\left(e_{i_{1}}, \ldots, e_{i_{m}}\right)$, then

$$
\begin{aligned}
& I_{\max }^{k}\left(e_{i_{1}}, \ldots, e_{i_{m}}\right)-I^{h}\left(x, V(\gamma)^{*}\right) \\
& =\#\left\{p \in(\operatorname{Ext} \gamma)^{*} / \begin{array}{l}
\text { the plaquette } p \text { contains in its boundary } \\
\text { two links of } V(\gamma)^{*} \text { incident to } x
\end{array}\right\}
\end{aligned}
$$

(recall that $\left.(\operatorname{Ext} \gamma)^{*} \equiv \mathbb{L}^{*} \backslash V(\gamma)^{*}\right)$.

This remark will be will be used only in the second step where we shall estimate $\left.\mid H_{1} V(\gamma)^{*}\right) \mid$. We now list the values of $I_{\max }^{h}(m)$ and $I_{\min }^{h}(m)\left(I_{\min }^{h}(m)\right.$ is obviously defined) obtained by investigating all possibilities with the constraints described above.

\begin{tabular}{llll}
\hline$m$ & $I_{\min }^{h}(m)$ & $I_{\max }^{h}(m)$ & $4 m-I_{\max }^{\text {h }}(m)-8$ \\
\hline 3 & 3 & 3 & 1 \\
4 & 4 & 6 & 2 \\
5 & 5 & 9 & 3 \\
6 & 7 & 13 & 3 \\
7 & 7 & 18 & 2 \\
8 & 8 & 24 & 0 \\
\hline
\end{tabular}

We observe that for any $\gamma$ and any $x$ in $\mathscr{S}_{m}\left(V(\gamma)^{*}\right)$ it is $4 m-I^{h}\left(x, V(\gamma)^{*}\right)-8 \geqq 1$, unless $m=8$ and $I^{n}\left(x, V(\gamma)^{*}\right)=24$. This concludes the proof.

Second step: We shall now estimate the term $\left|H_{1}\left(V(\gamma)^{*}\right)\right|$. This number will be estimated below through the knowledge of a certain set of plaquettes of $(\operatorname{Ext} \gamma)^{*}$. For any contour $\gamma$ we let $\mathscr{S}\left(\gamma^{*}\right)$ be the set of sites $x$ in $V(\gamma)^{*}$ such that if $\left[\gamma^{*}, x\right] \in\left[\gamma^{*}, x\right]\left(e_{i_{1}}, \ldots, e_{i_{m}}\right)$ then $I^{k}\left(x, V(\gamma)^{*}\right) \neq I_{\text {max }}^{k}\left(e_{i_{1}}, \ldots, e_{i_{m}}\right)$.

We denote: $J^{h}(x)=I_{\max }^{h}\left(e_{i_{1}}, \ldots, e_{i_{m}}\right)-I^{h}\left(x, V(\gamma)^{*}\right)$.

If $x \in \mathscr{S}\left(\gamma^{*}\right)$ we let $\mathscr{P}_{*}(x)$ be the set of plaquettes in $(\text { Ext } \gamma)^{*}$ which contains two links of $V(\gamma)^{*}$ incident to $x$ and $\mathscr{P}_{*} \equiv \bigcup_{x \in \mathscr{P}\left(\gamma^{*}\right)} \mathscr{P}_{*}(x)$. This set divide in the following subsets (see Fig. B.2): 


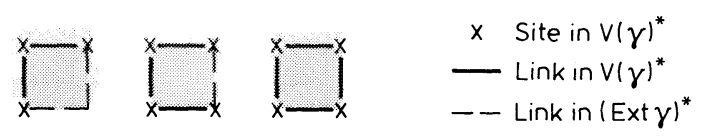

Fig. B.2. The different plaquettes of $\mathscr{P}_{*}$ : the indicated sites belong to $\mathscr{S}\left(\gamma^{*}\right)$

$-\mathscr{P}_{*}^{[4]}$ be the set of plaquettes in $(E x t \gamma)^{*}$ which contain four links and four sites of $V(\gamma)^{*}$

- $\mathscr{P}_{*}^{[3]}$ be the set of plaquettes in $(E x t \gamma)^{*}$ which contain three links of $V(\gamma)^{*}$ one link in $(\operatorname{Ext} \gamma)^{*}$ and four sites of $V(\gamma)^{*}$

$-\mathscr{P}_{*}^{[2]}$ be the set of plaquettes in $(\text { Ext } \gamma)^{*}$ which contain two links of $V(\gamma)^{*}$ incident to a site of $\mathscr{S}\left(\gamma^{*}\right)$ and two links in $(\operatorname{Ext} \gamma)^{*}$.

We denote $P_{n}, n=2,3,4$, the cardinality of the set $\mathscr{P}_{*}^{[n]}$. Under these notations we have the following

\section{Lemma B.4.}

$$
\begin{aligned}
& \left|H_{1}\left(V(\gamma)^{*}\right)\right| \leqq q^{\frac{1}{10}{ }_{x \in \mathscr{G}} J^{J^{h}(x)}}=q^{\frac{1}{10}\left(P_{2}+2 P_{3}+4 P_{4}\right)} \\
& \leqq q^{\frac{1}{10} \sum_{x \in V(\gamma)^{*}}\left[4 I^{\ell}\left(x, V(\gamma)^{*}\right)-I^{h}\left(x, V(\gamma)^{*}-8\right]\right.} .
\end{aligned}
$$

Proof. We first notice that since a closed complex is $p$-torsion free except for $0=1$, then it follows from (A.8)

$$
\left|H_{1}\left(V(\gamma)^{*}\right)\right| \leqq q^{\pi^{1}\left(V(\gamma)^{*}\right)+\tau^{1}\left(V(\gamma)^{*}\right)} .
$$

Next we recall that for any 1-chain of the complex $V(\gamma)^{*}$ may be written in terms of a $\partial$-basis

$$
\begin{gathered}
\left\{a_{i} \mid i=1 \ldots v^{0}\right\}, \quad\left\{x_{i} \mid i=1 \ldots \tau^{0}\right\}, \quad\left\{h_{i} \mid i=1 \ldots \pi^{1}\right\}, \\
\left\{b_{i} \mid i=1 \ldots v^{1}\right\}, \quad \text { and } \quad\left\{t_{i} \mid i=1 \ldots \tau^{1}\right\}
\end{gathered}
$$

(cf. Appendix A.3), where $h_{i}$ and $t_{i}$ are 1-cycles, nevertheless they are not boundary of 2-chains in $V(\gamma)^{*}$.

Thus to compute $\pi^{1}\left(V(\gamma)^{*}\right)+\tau^{1}\left(V(\gamma)^{*}\right)$ we shall estimate the maximal number of linearly independent cycles that are not boundaries of 2-cycles in $V(\gamma)^{*}$. Let us denote $\mathscr{B}=\left\{c_{i}\right\}_{i=1, \ldots, \pi^{1}+\tau^{1}}$ the family of these cycles. Since $V(\gamma)^{*}$ is closed the family $\mathscr{B}$ can be chosen in a such way that the cycles $c_{i}$ take non-vanishing values only on the boundary of $V(\gamma)^{*}$. Each one of the cycles in $\mathscr{B}$ is the boundary of a 2-chain constituted of plaquettes in $(\operatorname{Ext} \gamma)^{*}$ (i.e. a 2-chain non-vanishing on these plaquettes).

As an example we show in Fig. B.3 three kinds of 1-cycle.

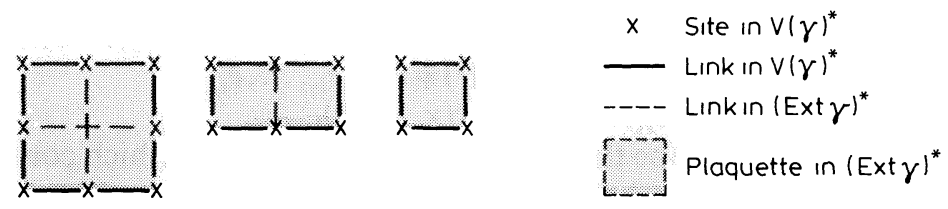

Fig. B.3. Non-bounding 1-cycles in $V(\gamma)^{*}$ 
We observe that for any of these cycles 2-chain exists a necessary in $(\operatorname{Ext} \gamma)^{*}$ non-vanishing on $\mathscr{P}_{*}$. Moreover there exist sites of $\mathscr{S}$ such that the cycle is nonvanishing on the links which are in the coboundary of these sites and in the boundary of a plaquette of $\mathscr{P}_{*}$.

Now from Theorem A.1 we deduce that

$$
\begin{aligned}
H^{1}\left(V(\gamma)^{*}\right) & =H_{2}(\operatorname{Ext} \gamma), \\
\pi^{1}\left(V(\gamma)^{*}\right) & =\pi^{2}(\operatorname{Ext} \gamma), \\
\tau^{1}\left(V(\gamma)^{*}\right) & =\tau^{1}(\operatorname{Ext} \gamma) .
\end{aligned}
$$

This isomorphism shows that to each 1-cycle in $\mathscr{B}$ it corresponds a 2-cycle in Ext $\gamma$. We shall denote $\mathscr{B}^{\prime}=\left\{c_{i}^{\prime}\right\}_{i=1, \ldots, \pi^{1}+\tau^{1}}$ the family of such linearly independent 2-cycles. Furthermore the dual of a plaquette $p$ of $\mathscr{P}_{*}^{[n]} \subset \mathscr{P}_{*}$ is a plaquette $p^{\prime}$ of $\left(\mathscr{P}_{*}^{[n]}\right)^{*} \subset\left(\mathscr{P}_{*}\right)^{*} \subset$ Ext $\gamma$. This plaquette $p^{\prime}$ contains in its coboundary $n$ cubes $(n \leqq 4)$ of $V(\gamma)$, the other $(4-n)$ cubes belongs to Ext $\gamma$. Hereafter we shall denote $\left(\mathscr{P}_{*}^{[n]}\right)^{*}$ as $\mathscr{P}^{[n]}$ and $\left(\mathscr{P}_{*}\right)^{*}$ as $\mathscr{P}$. The 2-cycles in $\mathscr{B}^{\prime}$ are not boundaries of 3-chains in Ext $\gamma$ but boundaries of 3-chains in $V(\gamma)$ and necessary non-vanishing on the set of cubes in the coboundary of plaquettes of $\mathscr{P}$.

Since a cube of $V(\gamma)$ cannot contains five plaquettes of $V(\gamma)$ and one plaquette of Ext $\gamma$ (see Remark B.1) it follows that any 2-cycle is a boundary of a 3-chain nonvanishing on at least four cubes of $V(\gamma)$. Thus this 2-cycle is non-vanishing on at least 16 plaquettes of Ext $\gamma$ as indicated in Fig. B.4, which corresponds to a situation where only the indicated sixteen plaquettes are ordered and all other plaquettes are disordered. In this case the dual of each plaquette belongs to $\mathscr{P}^{[4]}$. Denoting $c^{\prime}$ the considered 2-cycle one observes that there exist 40 sites of $\mathscr{S}$ such that $c^{\prime}$ do not vanish on plaquettes dual to plaquettes of $\mathscr{P}_{*}=\bigcup_{x \in \mathscr{S}} \mathscr{P}_{*}(x)$.

Clearly for any 2-cycle $c^{\prime}$ at least 40 sites of $\mathscr{S}$ would exist such that $c^{\prime}\left(p^{\prime}\right) \neq 0$ for $p^{\prime} \in \mathscr{P}$ dual to a plaquette, $p \in \mathscr{P}_{*}=\bigcup_{x \in \mathscr{S}} \mathscr{P}_{*}(x)$.

Since each plaquette, $p^{\prime}$, of $\mathbb{L}$ contains at most four cubes in its coboundary one may choose the family $\mathscr{B}^{\prime}$ in a such way that there exist at most four 2-cycles of this family which do not vanish on a plaquette $p^{\prime} \in \mathscr{P}$.

Recalling that a site $x$ of $\mathscr{S}$ contains in its coboundary $J^{h}(x)$ plaquettes of $\mathscr{P}_{*}(x), 4 J^{k}(x)$ is therefore the maximal number of 2-cycles non-vanishing on plaquettes in $\mathscr{P}$ dual to plaquettes in $\mathscr{P}_{*}(x)$. Therefore we get

$$
\pi^{1}\left(V(\gamma)^{*}\right)+\tau^{1}\left(V(\gamma)^{*}\right) \leqq \frac{1}{40} \sum_{x \in \mathscr{S}} 4 J^{\natural}(x)
$$

which imply the first inequality in Lemma B.4.
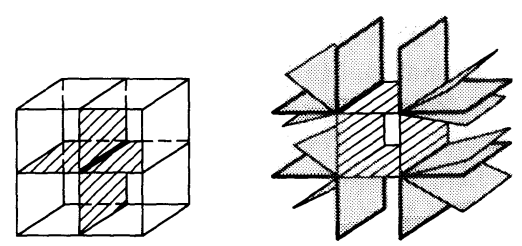

Fig. B.4. A non-bounding 2-cycle (non-hatched plaquettes) in $V(\gamma)$ and its dual (grey plaquettes) 
To prove the equality of this lemma we notice that it is a simple consequence of the definitions of $J^{h}(x)$ and $\mathscr{P}_{*}^{[n]}$.

To prove the second inequality in the lemma we first notice that:

$$
\begin{gathered}
\sum_{x \in V(\gamma)^{*}}\left[4 I^{\ell}\left(x, V(\gamma)^{*}\right)-I^{h}\left(x, V(\gamma)^{*}\right)-8\right]-\sum_{x \in \mathscr{S}} J^{h}(x) \\
\geqq \sum_{m=3}^{8} \sum_{x \in \mathscr{S}_{m}\left(V(\gamma)^{*}\right)}\left[4 m-I^{\not h}\left(x, V(\gamma)^{*}\right)-J^{h}(x)-8\right],
\end{gathered}
$$

since $S \subset V(\gamma)^{*}$. It follows from Remark B.2 that for $x \in \bigcup_{3 \leqq m \leqq 7} \mathscr{S}_{m}$ it is $I^{k}\left(x, V(\gamma)^{*}\right)$ $+J^{h}(x) \leqq I_{\max }^{p}(m)$, and for $x \in \mathscr{S}_{8}$ it is $I^{h}\left(x, V(\gamma)^{*}\right)+I^{h}\left(x, \mathscr{P}_{*}^{m}\right)=24$. Since

$$
\sum_{m=3}^{8} \sum_{\mathscr{S}_{m}\left(V(\gamma)^{*}\right)}\left[4 m-I_{\max }^{h}(m)-8\right]=S_{3}+2 S_{4}+3 S_{5}+3 S_{6}+2 S_{7} \geqq 0
$$

with $S_{m}$ the cardinality of $\mathscr{S}_{m}$ (see the proof of Lemma B.3), we get the desired inequality. This ends the proof.

Lemma 4.3 follows from Lemmas B.1, B.2, B.4, and B.3.

Acknowledgements. We are indebted to J. Bricmont, C.-E. Pfister, and S. Miracle-Solé for very helpful discussions. We thank C.-E. Pfister and the referee for very useful remarks and improvements. L. Laanait is grateful to the Faculté des Sciences de Luminy and CPT for financial support and kind hospitality.

\section{References}

1. Kotecky, R., Shlosman, S.B.: Commun. Math. Phys. 83, 493 (1982)

2. Fröhlich, J., Israel, R., Lieb, E., Simon, B.: Commun. Math. Phys. 62, 1 (1978); J. Stat. Phys. 22, 297 (1978)

3. Laanait, L., Messager, A., Ruiz, J.: Commun. Math. Phys. 105, 527 (1986)

4. Bricmont, J., Kuroda, K., Lebowitz, J.L.: Commun. Math. Phys. 101, 501 (1985)

5. Dinaburg, E.I., Sinaï, Ya.G.: Tr. Conf. Mat. Fiz., Dubna (1984)

6. Martirosian, D.G.: Commun. Math. Phys. 105, 281 (1986)

7. Kotecky, R., Laanait, L., Messager, A., Ruiz, J.: The standard Pirogov-Sinai theory in the $q$-state Potts model: topological aspect. Preprint CPT-Marseille

8. Kogut, J.B.: Phys. Rev. D 21, 2316 (1980)

9. Wilson, K.: Phys. Rev. D 10, 2445 (1974)

10. Seiler, E.: Gauge theories as a problem of constructive quantum field theory and statistical mechanics. Lecture Notes in Physics, Vol. 159. Berlin, Heidelberg, New York: Springer 1982

11. Gallavotti, G., Guerra, F., Miracle-Solé, S.: Lecture Notes in Physics, Vol. 80. Berlin, Heidelberg, New York: Springer 1978

12. Borgs, C.: Commun. Math. Phys. 96, 251 (1984)

13. Allexandroff, P.: Combinatorial topology, Vols. 1-3. Rochester, New York: Graylock 1956

14. Guth, A.H.: Phys. Rev. D 21, 2291 (1980)

15. Fröhlich, J., Spencer, T.: Commun. Math. Phys. 83, 411 (1982)

16. King, C.: Commun. Math. Phys. 105, 675 (1986)

17. Creutz, M., Jacobs, L., Rebbi, C.: Phys. Rev. Lett. 42, 1390 (1980)

18. Jongeward, G.A., Stack, J.D., Jayaprakash, C.: Phys. Rev. D 21, 3360 (1980)

19. Ginsparg, P., Goldschmidt, Y.Y., Zuber, J.-B.: Nucl. Phys. B 170 FS1, 409 (1980)

20. Aizenman, M., Fröhlich, J.: Nucl. Phys. B 235 FS11, 1 (1980)

21. Pfister, C.-E.: Commun. Math. Phys. 86, 375 (1982) 
22. Ginibre, J.: Commun. Math. Phys. 16, 310 (1970)

Messager, A., Miracle-Solé, S., Pfister, C.-E.: Commun. Math. Phys. 59, 1929 (1978)

23. Gallavotti, G., Martin-Löf, A., Miracle-Sole, S.: Some problems connected with the description of coexisting phases at low temperatures in the Ising model. In Lecture Notes in Physics, Vol. 20. Berlin, Heidelberg, New York: Springer 1973

24. Kotecky, R., Preiss, D.: Commun. Math. Phys. 103, 491 (1986)

25. Gallavotti, G., Miracle-Solé, S.: Phys. Rev. B 5, 2555 (1980)

26. Zinove'v, Y.M.: Theor. Math. Phys. 43, 481 (1980)

27. Druhl, K., Wagner, H.: Ann. Phys. 141, 225 (1982)

28. Martirosian, D.G.: Sov. J. Contemp. Math. Anal. 14, 19 (1979)

29. Aizenman, M., Chayes, J.T., Chayes, L., Fröhlich, J., Russo, L.: Commun. Math. Phys. 92,19 (1983)

30. Kesten, H.: Adv. Appl. Prob. 12, 848 (1980). Commun. Math. Phys. 74, 41 (1980)

31. Russo, L.: Wahrscheinlichkeitstheorie Verw. Geb. 56, 229 (1981)

32. Aizenman, M., Barsky, D.J.: Commun. Math. Phys. 108, 489 (1987)

Communicated by K. Gawedzki

Received January 29, 1987; in revised form April 5, 1989 
